\title{
Stability analysis of general humoral immunity HIV dynam- ics models with discrete delays and HAART
}

\author{
A. M. Elaiw ${ }^{a, *}$, E. Kh. Elnahary \\ ${ }^{a}$ Department of Mathematics, Faculty of Science, King Abdulaziz University, P. O. Box 80203, Jeddah 21589, Saudi Arabia. \\ ${ }^{b}$ Department of Mathematics, Faculty of Science, Sohag University, Sohag, Egypt.
}

\begin{abstract}
We investigate a general HIV infection model with three types of infected cells: latently infected cells, long-lived productively infected cells, and short-lived productively infected cells. We consider two kinds of target cells: CD4 ${ }^{+} \mathrm{T}$ cells and macrophages. We incorporate three discrete time delays into the model. Moreover, we consider the effect of humoral immunity on the dynamical behavior of the HIV. The HIV-target incidence rate, production/proliferation, and removal rates of the cells and HIV are represented by general nonlinear functions. We show that the solutions of the proposed model are nonnegative and ultimately bounded. We derive two threshold parameters which determine the stability of the three steady states of the model. Using Lyapunov functionals, we established the global stability of the steady states of the model. The theoretical results are confirmed by numerical simulations.
\end{abstract}

Keywords: HIV infection, global stability, humoral immune response, latency, viral reservoirs.

2010 MSC: 34D20, 34D23, 37N25, 92B05.

(C)2018 All rights reserved.

\section{Introduction}

During the last decades several mathematical models have been proposed with the aim to understand the dynamics of HIV in the human body [1, 3-8, 10-12, 14, 16-18, 20-25, 27, 28, 30-33, 35-37, 39]. The basic and pioneering model describing the HIV dynamics is due Nowak and Bangham [33]. The model contains three compartments: uninfected $\mathrm{CD}^{+} \mathrm{T}$ cells (s), infected cells (y), and free HIV particles (p):

$$
\begin{aligned}
\dot{s}(t) & =\rho-\delta s(t)-\bar{\lambda} s(t) p(t), \\
\dot{y}(t) & =\bar{\lambda} s(t) p(t)-\eta y(t), \\
\dot{p}(t) & =\bar{N} \eta y(t)-g p(t),
\end{aligned}
$$

where $\rho, \delta$, and $\bar{\lambda}$ represent the production, death, and infection rates of the uninfected CD4 ${ }^{+} \mathrm{T}$ cells, respectively; $\eta$ and $g$ are the death rate constants of the infected cells and free HIV, respectively; $\bar{N}$ is the

\footnotetext{
*Corresponding author

Email address: a_m_elaiw@yahoo.com (A. M. Elaiw)
}

doi: $10.22436 /$ jmcs.018.04.05

Received: 2015-12-24 Revised: 2018-11-19 Accepted: 2018-11-22 
average number of HIV particles generated in the lifetime of the infected cells. A lot of considerations have been added that aim to get the best representation of the HIV infection. Most notably is latent HIV reservoirs which serve as a major barrier in curing HIV infection. In despite of antiretroviral therapy limits significantly the level of HIV in the blood, there still a low viral load due to ongoing latently infected cells reservoir reactivation. Variant models have been developed to study the dynamics of HIV in the presence of latent reservoirs (see, e.g., $[2,9,13,15,29])$.

In a very recent work Elaiw et al. [19] have modified model (1.1) by considering: (i) three types of infected cells, latently infected cells $(w)$, short-lived productively infected cells (y), and long-lived productively infected cells (u); (ii) three types of time delays; (iii) antibody immune response (x); (iv) general nonlinear functions for the HIV-target incidence rate, production/proliferation and removal rates of the cells and HIV; and (v) highly active antiretroviral therapy (HAART) which combine both reverse transcriptase inhibitor and protease inhibitor. The model presented in [19] is given by:

$$
\begin{aligned}
\dot{s}(t) & =\pi(s(t))-\left(1-\varepsilon_{1}\right) \bar{\lambda} \chi(s(t), p(t)), \\
\dot{w}(t) & =\left(1-\varepsilon_{1}\right) \bar{\lambda}_{1} e^{-\mu_{1} \tau_{1}} \chi\left(s\left(t-\tau_{1}\right), p\left(t-\tau_{1}\right)\right)-(\alpha+\beta) \psi_{1}(w(t)), \\
\dot{y}(t) & =\left(1-\varepsilon_{1}\right) \bar{\lambda}_{2} e^{-\mu_{2} \tau_{2}} \chi\left(s\left(t-\tau_{2}\right), p\left(t-\tau_{2}\right)\right)+\alpha \psi_{1}(w(t))-\eta \psi_{2}(y(t)), \\
\dot{u}(t) & =\left(1-\varepsilon_{1}\right) \bar{\lambda}_{3} e^{-\mu_{3} \tau_{3}} \chi\left(s\left(t-\tau_{3}\right), p\left(t-\tau_{3}\right)\right)-v \psi_{3}(u(t)), \\
\dot{p}(t) & =\left(1-\varepsilon_{2}\right) \bar{N} \eta \psi_{2}(y(t))+\left(1-\varepsilon_{2}\right) \bar{M} v \psi_{2}(u(t))-g \psi_{4}(p(t))-\mu \psi_{4}(p(t)) \psi_{5}(x(t)), \\
\dot{x}(t) & =r \psi_{4}(p(t)) \psi_{5}(x(t))-\omega \psi_{5}(x(t)) .
\end{aligned}
$$

Parameter $\tau_{1}$ is the time between viral entry and latent infection (i.e., the integration of viral DNA into cell's DNA has finished), while $\tau_{2}$ and $\tau_{3}$ are the times between viral entry and viral production from short-lived productively infected and long-lived productively infected cells, respectively. The factor $e^{-\mu_{j} \tau_{j}}, j=1,2,3$ accounts for the loss of target cells during the delay period of length $\tau_{j}$, where $\mu_{j}>0$ is constant. The model incorporates reverse transcriptase inhibitor (RTI) with efficacy $\varepsilon_{1}$ and protease inhibitor (PI) with efficacy $\varepsilon_{2}$, where $\varepsilon_{1}, \varepsilon_{2} \in[0,1] . \chi, \pi, \psi_{j}, j=1, \ldots, 5$ are general nonlinear functions.

Model (1.2) has considered the interaction of the HIV with one class of target cells, CD4 ${ }^{+} \mathrm{T}$ cells. It has been reported in [34] that the HIV can infect both the $\mathrm{CD}^{+} \mathrm{T}$ cells and macrophages. To have more accurate HIV dynamics model the interaction between the HIV with the macrophages have to be considered. The aim of this paper is to propose and analyze an HIV infection model which improves model (1.2) by taking into account two classes of target cells, $\mathrm{CD}^{+} \mathrm{T}$ cells and macrophages. We propose the following model:

$$
\begin{aligned}
\dot{s}_{i}(t)= & \pi_{i}\left(s_{i}(t)\right)-\lambda_{i} \chi_{i}\left(s_{i}(t), p(t)\right), \\
\dot{w}_{i}(t)= & \lambda_{1 i} e^{-\mu_{1 i} \tau_{1 i}} \chi_{i}\left(s_{i}\left(t-\tau_{1 i}\right), p\left(t-\tau_{1 i}\right)\right)-\left(\alpha_{i}+\beta_{i}\right) \psi_{1 i}\left(w_{i}(t)\right), \\
\dot{y}_{i}(t)= & \lambda_{2 i} e^{-\mu_{2 i} \tau_{2 i}} \chi_{i}\left(s_{i}\left(t-\tau_{2 i}\right), p\left(t-\tau_{2 i}\right)\right)+\alpha_{i} \psi_{1 i}\left(w_{i}(t)\right)-\eta_{i} \psi_{2 i}\left(y_{i}(t)\right), \\
\dot{u}_{i}(t)= & \lambda_{3 i} e^{-\mu_{3 i} \tau_{3 i}} \chi_{i}\left(s_{i}\left(t-\tau_{3 i}\right), p\left(t-\tau_{3 i}\right)\right)-v_{i} \psi_{3 i}\left(u_{i}(t)\right), \\
\dot{p}(t)= & \sum_{i=1}^{2}\left(N_{i} \eta_{i} e^{-\mu_{4 i} \tau_{4 i}} \psi_{2 i}\left(y_{i}\left(t-\tau_{4 i}\right)\right)+M_{i} \nu_{i} e^{-\mu_{5 i} \tau_{5 i}} \psi_{3 i}\left(u_{i}\left(t-\tau_{5 i}\right)\right)\right) \\
& -g \psi_{41}(p(t))-\mu \psi_{41}(p(t)) \psi_{42}(x(t)), \\
\dot{x}(t)= & r \psi_{41}(p(t)) \psi_{42}(x(t))-\omega \psi_{42}(x(t)),
\end{aligned}
$$

where $i=1$ for the $\mathrm{CD} 4^{+} \mathrm{T}$ cells and $i=2$ for the macrophages. We have $\lambda_{\mathrm{m} 1}=\left(1-\varepsilon_{1}\right) \bar{\lambda}_{\mathrm{m} 1}, \lambda_{\mathrm{m} 2}=$ $\left(1-f \varepsilon_{1}\right) \bar{\lambda}_{m 2}, m=1,2,3, N_{1}=\left(1-\varepsilon_{2}\right) \bar{N}_{1}, M_{1}=\left(1-\varepsilon_{2}\right) \bar{M}_{1}, N_{2}=\left(1-h \varepsilon_{2}\right) \bar{N}_{2}, M_{2}=\left(1-h \varepsilon_{2}\right) \bar{M}_{2}$, $\lambda_{i}=\lambda_{1 i}+\lambda_{2 i}+\lambda_{3 i}$ and $f, h \in(0,1)$. The parameters $\tau_{4 i}$ and $\tau_{5 i}$ represent the time necessary for producing new infectious viruses from the short-lived productively infected and long-lived productively infected cells, respectively. All the parameters are positive.

Functions $\chi_{i}, \pi_{i}, \psi_{j i}, j=1, \ldots, 4, i=1,2$, are continuously differentiable and satisfy the following hypotheses: 
H1 (i) there exists $s_{\mathfrak{i}}^{0}$ such that $\pi_{\mathfrak{i}}\left(s_{\mathfrak{i}}^{0}\right)=0, \pi_{\mathfrak{i}}\left(s_{\mathfrak{i}}\right)>0$ for $s_{\mathfrak{i}} \in\left[0, s_{\mathfrak{i}}^{0}\right)$;

(ii) $\pi_{i}^{\prime}\left(s_{i}\right)<0$ for $s_{i} \in(0, \infty)$;

(iii) there are $b_{i}>0$ and $\bar{b}_{i}>0$ such that $\pi_{i}\left(s_{i}\right) \leqslant b_{i}-\bar{b}_{i} s_{i}$ for $s_{i} \in[0, \infty)$;

$\mathrm{H} 2 \quad$ (i) $\chi_{i}\left(s_{i}, p\right)>0$ and $\chi_{i}(0, p)=\chi_{i}\left(s_{i}, 0\right)=0$ for $s_{i}, p \in(0, \infty)$;

(ii) $\frac{\partial x_{i}\left(x_{i}, p\right)}{\partial s_{i}}>0, \frac{\partial x_{i}\left(s_{i}, p\right)}{\partial p}>0$ and, $\frac{\partial x_{i}\left(s_{i}, 0\right)}{\partial p}>0$ for all $s_{i}, p \in(0, \infty)$;

(iii) $\frac{d}{d s_{i}}\left(\frac{\partial \chi_{i}\left(s_{i}, 0\right)}{\partial p}\right)>0$ for $s_{i} \in(0, \infty)$;

$\mathrm{H} 3 \quad$ (i) $\psi_{j i}(\eta)>0$ for $\eta \in(0, \infty), \psi_{j i}(0)=0, j=1, \ldots, 4, i=1,2$;

(ii) $\psi_{j i}^{\prime}(\eta)>0, \psi_{42}^{\prime}(\eta)>0$ for $\eta \in(0, \infty), j=1,2,3, i=1,2, \psi_{41}^{\prime}(\eta)>0$, for $\eta \in[0, \infty)$;

(iii) there are $\alpha_{j i}>0, j=1, \ldots, 4, i=1,2$, such that, $\psi_{j i}(\eta) \geqslant \alpha_{j i} \eta$ for $\eta \in[0, \infty)$;

$\mathrm{H} 4 \frac{\chi_{i}\left(s_{i}, p\right)}{\psi_{41}(p)}$ is decreasing function w.r.t $p$ for $p \in(0, \infty)$.

Remark 1.1. From H1-H4 we have

$$
\left(\frac{\chi_{i}\left(s_{i}, p\right)}{\psi_{41}(p)}-\frac{\chi_{i}\left(s_{i}, p^{*}\right)}{\psi_{41}\left(p^{*}\right)}\right)\left(\chi_{i}\left(s_{i}, p\right)-\chi_{i}\left(s_{i}, p^{*}\right)\right) \leqslant 0,
$$

which gives

$$
\left(\frac{\chi_{i}\left(s_{i}, p\right)}{\chi_{i}\left(s_{i}, p^{*}\right)}-\frac{\psi_{41}(p)}{\psi_{41}\left(p^{*}\right)}\right)\left(1-\frac{\chi_{i}\left(s_{i}, p^{*}\right)}{\chi_{i}\left(s_{i}, p\right)}\right) \leqslant 0 .
$$

We consider system (1.3) with the initial conditions:

$$
\begin{aligned}
s_{1}(t) & =\varphi_{1}(\theta), \quad s_{2}(t)=\varphi_{2}(\theta), \quad w_{1}(t)=\varphi_{3}(\theta), \quad w_{2}(t)=\varphi_{4}(\theta), \quad y_{1}(t)=\varphi_{5}(\theta), \\
y_{2}(t) & =\varphi_{6}(\theta), \quad u_{1}(t)=\varphi_{7}(\theta), \quad u_{2}(t)=\varphi_{8}(\theta), \quad p(t)=\varphi_{9}(\theta), \quad x(t)=\varphi_{10}(\theta), \\
\varphi_{j}(\theta) & \geqslant 0, \quad \theta \in[-\sigma, 0], \quad j=1, \ldots, 10,
\end{aligned}
$$

where $\sigma=\max \left\{\tau_{11}, \tau_{12}, \tau_{21}, \tau_{22}, \tau_{31}, \tau_{32}, \tau_{41}, \tau_{42}, \tau_{51}, \tau_{52}\right\}$ and denote by $C$ is the Banach space of continuous functions mapping the interval $[-\sigma, 0]$ into $\mathbb{R}_{\geqslant 0}$ and $\left(\varphi_{1}(\theta), \ldots, \varphi_{10}(\theta)\right) \in C\left([-\sigma, 0], \mathbb{R}_{\geqslant 0}^{10}\right)$. Then, the uniqueness of the solution for $t>0$ is guaranteed [26].

\subsection{Preliminaries}

Lemma 1.2. Let hypotheses H1-H3 be valid, then the solutions of system (1.3) is non-negative and ultimately bounded.

Proof. Let us write system (1.3) in matrix form $\dot{k}(t)=L(k(t))$, where $k=\left(s_{1}, s_{2}, w_{1}, w_{2}, y_{1}, y_{2}, u_{1}, u_{1}, p, x\right)^{\top}$, $\mathrm{L}=\left(\mathrm{L}_{1}, \mathrm{~L}_{2}, \ldots, \mathrm{L}_{10}\right)^{\mathrm{T}}$ and

$$
\begin{aligned}
& L(k(t))=\left(\begin{array}{c}
L_{1}(k(t)) \\
L_{2}(k(t)) \\
\vdots \\
L_{10}(k(t))
\end{array}\right), \\
& L=\left(\begin{array}{c}
\pi_{1}\left(s_{1}(t)\right)-\lambda_{1} \chi_{1}\left(s_{1}(t), p(t)\right) \\
\pi_{2}\left(s_{2}(t)\right)-\lambda_{2} \chi_{2}\left(s_{2}(t), p(t)\right) \\
\lambda_{11} e^{-\mu_{11} \tau_{11}} \chi_{1}\left(s_{1}\left(t-\tau_{11}\right), p\left(t-\tau_{11}\right)\right)-\left(\alpha_{1}+\beta_{1}\right) \psi_{11}\left(w_{1}(t)\right) \\
\lambda_{12} e^{-\mu_{12} \tau_{12}} \chi_{2}\left(s_{2}\left(t-\tau_{12}\right), p\left(t-\tau_{12}\right)\right)-\left(\alpha_{2}+\beta_{2}\right) \psi_{12}\left(w_{2}(t)\right) \\
\lambda_{21} e^{-\mu_{21} \tau_{21}} \chi_{1}\left(s_{1}\left(t-\tau_{21}\right), p\left(t-\tau_{21}\right)\right)+\alpha_{1} \psi_{11}\left(w_{1}(t)\right)-\eta_{1} \psi_{21}\left(y_{1}(t)\right) \\
\lambda_{22} e^{-\mu_{22} \tau_{22}} \chi_{2}\left(s_{2}\left(t-\tau_{22}\right), p\left(t-\tau_{22}\right)\right)+\alpha_{2} \psi_{12}\left(w_{2}(t)\right)-\eta_{2} \psi_{22}\left(y_{2}(t)\right) \\
\lambda_{31} e^{-\mu_{31} \tau_{31}} \chi_{1}\left(s_{1}\left(t-\tau_{31}\right), p\left(t-\tau_{31}\right)\right)-v_{1} \psi_{31}\left(u_{1}(t)\right) \\
\lambda_{32} e^{-\mu_{32} \tau_{32}} \chi_{2}\left(s_{2}\left(t-\tau_{32}\right), p\left(t-\tau_{32}\right)\right)-v_{2} \psi_{32}\left(u_{2}(t)\right) \\
\sum_{i=1}^{2}\left(N_{i} \eta_{i} e^{-\mu_{4 i} \tau_{4 i}} \psi_{2 i}\left(y_{i}\left(t-\tau_{4 i}\right)\right)+M_{i} v_{i} e^{-\mu_{5 i} \tau_{5 i}} \psi_{3 i}\left(u_{i}\left(t-\tau_{5 i}\right)\right)-g \psi_{41}(p(t))\right. \\
-\mu \psi_{41}(p(t)) \psi_{42}(x(t)) \\
r \psi_{41}(p(t)) \psi_{42}(x(t))-\omega \psi_{42}(x(t))
\end{array}\right) .
\end{aligned}
$$


We have

$$
\left.\mathrm{L}_{j}(k(t))\right|_{k(t) \in \mathbb{R}_{>0}^{10}} \geqslant 0, \quad j=1, \ldots, 10 .
$$

Using lemma 2 in [38], the solutions of system (1.3) with the initial states (1.4) satisfy $k(t) \in \mathbb{R}_{\geqslant 0}^{10}$ for all $t \geqslant 0$. The non-negativity of the model's solution implies that $\lim _{t \rightarrow \infty} \sup s_{i}(t) \leqslant M_{1 i}$, where $M_{1 i}=\frac{b_{i}}{\bar{b}_{i}}$. Let

$$
\begin{aligned}
T_{i}(t)= & N_{i} e^{-\mu_{1 i} \tau_{1 i}} s_{i}\left(t-\tau_{1 i}\right)+N_{i} e^{-\mu_{2 i} \tau_{2 i}} s_{i}\left(t-\tau_{2 i}\right)+M_{i} e^{-\mu_{3 i} \tau_{3 i}} s_{i}\left(t-\tau_{3 i}\right) \\
& +N_{i} w_{i}(t)+N_{i} y_{i}(t)+M_{i} u_{i}(t)
\end{aligned}
$$

then

$$
\begin{aligned}
\dot{\mathrm{T}}_{i}(t)= & N_{i} e^{-\mu_{1 i} \tau_{1 i}}\left[\pi_{i}\left(s_{i}\left(t-\tau_{1 i}\right)\right)-\lambda_{i} \chi_{i}\left(s_{i}\left(t-\tau_{1 i}\right), p\left(t-\tau_{1 i}\right)\right)\right] \\
& +N_{i} e^{-\mu_{2 i} \tau_{2 i}}\left[\pi_{i}\left(s_{i}\left(t-\tau_{2 i}\right)\right)-\lambda_{i} \chi_{i}\left(s_{i}\left(t-\tau_{2 i}\right), p\left(t-\tau_{2 i}\right)\right)\right] \\
& +M_{i} e^{-\mu_{3 i} \tau_{3 i}}\left[\pi_{i}\left(s_{i}\left(t-\tau_{3 i}\right)\right)-\lambda_{i} \chi_{i}\left(s_{i}\left(t-\tau_{3 i}\right), p\left(t-\tau_{3 i}\right)\right)\right] \\
& +N_{i}\left[\lambda_{1 i} e^{-\mu_{1 i} \tau_{1 i}} \chi_{i}\left(s_{i}\left(t-\tau_{1 i}\right), p\left(t-\tau_{1 i}\right)\right)-\left(\alpha_{i}+\beta_{i}\right) \psi_{1 i}\left(w_{i}(t)\right)\right] \\
& +N_{i}\left[\lambda_{2 i} e^{-\mu_{2 i} \tau_{2 i}} \chi_{i}\left(s_{i}\left(t-\tau_{2 i}\right), p\left(t-\tau_{2 i}\right)\right)+\alpha_{i} \psi_{1 i}\left(w_{i}(t)\right)-\eta_{i} \psi_{2 i}\left(y_{i}(t)\right)\right] \\
& +M_{i}\left[\lambda_{3 i} e^{-\mu_{3 i} \tau_{3 i}} \chi_{i}\left(s_{i}\left(t-\tau_{3 i}\right), p\left(t-\tau_{3 i}\right)\right)-v_{i} \psi_{3 i}\left(u_{i}(t)\right)\right] \\
\leqslant & N_{i} e^{-\mu_{1 i} \tau_{1 i}}\left[b_{i}-\bar{b}_{i} s_{i}\left(t-\tau_{1 i}\right)\right]+N_{i} e^{-\mu_{2 i} \tau_{2 i}}\left[b_{i}-\bar{b}_{i} s_{i}\left(t-\tau_{2 i}\right)\right] \\
& +M_{i} e^{-\mu_{3 i} \tau_{3 i}}\left[b_{i}-\bar{b}_{i} s_{i}\left(t-\tau_{3 i}\right)\right]-N_{i} \beta_{i} \alpha_{1 i} w_{i}(t)-N_{i} \eta_{i} \alpha_{2 i} y_{i}(t)-M_{i} v_{i} \alpha_{3 i} u_{i}(t) \\
\leqslant & b_{i}\left(N_{i} e^{-\mu_{1 i} \tau_{1 i}}+N_{i} e^{-\mu_{2 i} \tau_{2 i}}+M_{i} e^{-\mu_{3 i} \tau_{3 i}}\right)-\sigma_{i}\left[N_{i} e^{-\mu_{1 i} \tau_{1 i}} s_{i}\left(t-\tau_{1 i}\right)\right. \\
& \left.+N_{i} e^{-\mu_{2 i} \tau_{2 i}} s_{i}\left(t-\tau_{2 i}\right)+M_{i} e^{-\mu_{3 i} \tau_{3 i}} s_{i}\left(t-\tau_{3 i}\right)+N_{i} w_{i}(t)+N_{i} y_{i}(t)+M_{i} u_{i}(t)\right] \\
\leqslant & b_{i}\left(2 N_{i}+M_{i}\right)-\sigma_{i} T_{i}(t),
\end{aligned}
$$

where $\sigma_{i}=\min \left\{\bar{b}_{i}, \beta_{i} \alpha_{1 i}, \eta_{i} \alpha_{2 i}, v_{i} \alpha_{3 i}\right\}$. Then $\lim _{t \rightarrow \infty} \sup _{i}(t) \leqslant \frac{b_{i}\left(2 N_{i}+M_{i}\right)}{\sigma_{i}}$. The non-negativity of the system's variables implies that

$$
\begin{aligned}
& \lim _{t \rightarrow \infty} \sup w_{i}(t) \leqslant \frac{b_{i}\left(2 N_{i}+M_{2 i}\right)}{N_{i} \sigma_{i}}=M_{2 i}, \\
& \lim _{t \rightarrow \infty} \sup y_{i}(t) \leqslant \frac{b_{i}\left(2 N_{i}+M_{i}\right)}{N_{i} \sigma_{i}}=M_{2 i}, \\
& \lim _{t \rightarrow \infty} \sup u_{i}(t) \leqslant \frac{b_{i}\left(2 N_{i}+M_{i}\right)}{M_{i} \sigma_{i}}=M_{3 i} .
\end{aligned}
$$

Moreover, we let $T_{3}(t)=p(t)+\frac{\mu}{r} x(t)$. Then

$$
\begin{aligned}
\dot{\mathrm{T}}_{3} & =\sum_{i=1}^{2}\left(N_{i} \eta_{i} e^{-\mu_{4 i} \tau_{4 i}} \psi_{2 i}\left(y_{i}\left(t-\tau_{4 i}\right)\right)+M_{i} v_{i} e^{-\mu_{5 i} \tau_{5 i}} \psi_{3 i}\left(u_{i}\left(t-\tau_{5 i}\right)\right)\right)-g \psi_{41}(p)-\frac{\mu \omega}{r} \psi_{42}(x) \\
& \leqslant \sum_{i=1}^{2}\left(N_{i} \eta_{i} e^{-\mu_{4 i} \tau_{4 i}} \psi_{2 i}\left(M_{2 i}\right)+M_{i} v_{i} e^{-\mu_{5 i} \tau_{5 i}} \psi_{3 i}\left(M_{3 i}\right)\right)-g \alpha_{41} p-\frac{\mu \omega}{r} \alpha_{42} x \\
& \leqslant \sum_{i=1}^{2}\left(N_{i} \eta_{i} \psi_{2 i}\left(M_{2 i}\right)+M_{i} v_{i} \psi_{3 i}\left(M_{3 i}\right)\right)-\sigma_{3} T_{3}(t)
\end{aligned}
$$

where $\sigma_{3}=\min \left\{g \alpha_{41}, \omega \alpha_{42}\right\}$. Hence,

$$
\lim _{t \rightarrow \infty} \sup T_{3}(t) \leqslant \sum_{i=1}^{2} \frac{\left(N_{i} \eta_{i} \psi_{2 i}\left(M_{2 i}\right)+M_{i} v_{i} \psi_{3 i}\left(M_{3 i}\right)\right)}{\sigma_{3}}=M_{41},
$$




$$
\begin{aligned}
& \lim _{t \rightarrow \infty} \sup p(t) \leqslant M_{41}, \\
& \lim _{t \rightarrow \infty} \sup x(t) \leqslant \frac{r M_{41}}{\mu}=M_{42} .
\end{aligned}
$$

Therefore, $s_{i}(t), w_{i}(t), y_{i}(t), u_{i}(t), p(t)$, and $x(t)$ are ultimately bounded.

According to Lemma 1.2, we can show that the region

$$
\begin{gathered}
\Omega=\left\{\left(s_{1}, s_{2}, w_{1}, w_{2}, y_{1}, y_{2}, u_{1}, u_{2}, p, x\right) \in C^{10}:\left\|s_{i}\right\| \leqslant M_{1 i},\left\|w_{i}\right\| \leqslant M_{2 i},\right. \\
\left.\left\|y_{i}\right\| \leqslant M_{2 i},\left\|u_{i}\right\| \leqslant M_{3 i},\|p\| \leqslant M_{41},\|x\| \leqslant M_{42}\right\},
\end{gathered}
$$

is positively invariant with respect to system (1.3).

\subsection{Steady states}

We define the basic reproduction number $\mathrm{R}_{0}$ of system (1.3) as follows:

$$
\mathrm{R}_{0}=\sum_{i=1}^{2} \frac{\rho_{i}}{\psi_{41}^{\prime}(0)} \frac{\partial x_{i}\left(s_{i}^{0}, 0\right)}{\partial p}
$$

The steady state of (1.3) satisfies the following equations:

$$
\begin{aligned}
& 0=\pi_{i}\left(s_{i}\right)-\lambda_{i} \chi_{i}\left(s_{i}, p\right), \\
& 0=\lambda_{1 i} e^{-\mu_{1 i} \tau_{1 i}} \chi_{i}\left(s_{i}, p\right)-\left(\alpha_{i}+\beta_{i}\right) \psi_{1 i}\left(w_{i}\right), \\
& 0=\lambda_{2 i} e^{-\mu_{2 i} \tau_{2 i}} \chi_{i}\left(s_{i}, p\right)+\alpha_{i} \psi_{1 i}\left(w_{i}\right)-\eta_{i} \psi_{2 i}\left(y_{i}\right), \\
& 0=\lambda_{3 i} e^{-\mu_{3 i} \tau_{3 i}} \chi_{i}\left(s_{i}, p\right)-v_{i} \psi_{3 i}\left(u_{i}\right), \\
& 0=\sum_{i=1}^{2}\left(N_{i} \eta_{i} e^{-\mu_{4 i} \tau_{4 i}} \psi_{2 i}\left(y_{i}\right)+M_{i} \nu_{i} e^{-\mu_{5 i} \tau_{5 i}} \psi_{3 i}\left(u_{i}\right)\right)-g \psi_{41}(p)-\mu \psi_{41}(p) \psi_{42}(x), \\
& 0=r \psi_{41}(p) \psi_{42}(x)-\omega \psi_{42}(x) .
\end{aligned}
$$

From Eq. (1.10) we have two possible solutions: $\psi_{42}(x)=0$ and $\psi_{41}(p)=\omega / r$. The first possibility $\psi_{42}(x)=0$ implies that $x=0$. H3 implies that $\psi_{j i}^{-1}, j=1, \ldots, 4, i=1,2$, exists, strictly increasing and $\psi_{j i}^{-1}(0)=0$. Let us define

$$
\begin{aligned}
& \Delta_{1 i}\left(s_{i}\right)=\psi_{1 i}^{-1}\left(\frac{\lambda_{1 i} e^{-\mu_{1 i} \tau_{1 i}}}{\lambda_{i}\left(\alpha_{i}+\beta_{i}\right)} \pi_{i}\left(s_{i}\right)\right), \\
& \Delta_{2 i}\left(s_{i}\right)=\psi_{2 i}^{-1}\left(\frac{\alpha_{i} \lambda_{1 i} e^{-\mu_{1 i} \tau_{1 i}}+\left(\alpha_{i}+\beta_{i}\right) \lambda_{2 i} e^{-\mu_{2 i} \tau_{2 i}}}{\lambda_{i} \eta_{i}\left(\alpha_{i}+\beta_{i}\right)} \pi_{i}\left(s_{i}\right)\right), \\
& \Delta_{3 i}\left(s_{i}\right)=\psi_{3 i}^{-1}\left(\frac{\lambda_{3 i} e^{-\mu_{3 i} \tau_{3 i}}}{\lambda_{i} v_{i}} \pi_{i}\left(s_{i}\right)\right), \Delta_{4}\left(s_{i}\right)=\psi_{41}^{-1}\left(\sum_{i=1}^{2} \frac{\rho_{i}}{\lambda_{i}} \pi_{i}\left(s_{i}\right)\right),
\end{aligned}
$$

where $\rho_{i}=\sum_{i=1}^{2} \frac{N_{i} e^{-\mu_{4 i} \tau_{4 i}}\left(\alpha_{i} \lambda_{1 i} e^{-\mu_{1 i} \tau_{1 i}}+\left(\alpha_{i}+\beta_{i}\right) \lambda_{2 i} e^{\left.-\mu_{2 i} \tau_{2 i}\right)}+M_{i} e^{-\mu_{3 i} \tau_{3 i}} e^{-\mu_{5 i} \tau_{5 i} \lambda_{3 i}\left(\alpha_{i}+\beta_{i}\right)}\right.}{g\left(\alpha_{i}+\beta_{i}\right)}$. It follows from Eqs. (1.5)-(1.9) that:

$$
w_{i}=\Delta_{1 i}\left(s_{i}\right), \quad y_{i}=\Delta_{2 i}\left(s_{i}\right), \quad u_{i}=\Delta_{3 i}\left(s_{i}\right), \quad p=\Delta_{4}\left(s_{i}\right) .
$$

Obviously, $\Delta_{1 i}\left(s_{i}\right), \Delta_{2 i}\left(s_{i}\right), \Delta_{3 i}\left(s_{i}\right), \Delta_{4}\left(s_{i}\right)>0$ for $s_{i} \in\left[0, s_{i}^{0}\right)$ and $\Delta_{1 i}\left(s_{i}^{0}\right)=\Delta_{2 i}\left(s_{i}^{0}\right)=\Delta_{3 i}\left(s_{i}^{0}\right)=\Delta_{4}\left(s_{i}^{0}\right)=0$, $i=1$, 2. From Eqs. (1.5), (1.11), and (1.12) we obtain

$$
\sum_{i=1}^{2} \rho_{i} \chi_{i}\left(s_{i}, \Delta_{4}\left(s_{i}\right)\right)-\psi_{41}\left(\Delta_{4}\left(s_{i}\right)\right)=0 .
$$

Eq. (1.13) has two possible solutions, $\Delta_{4}=0$ and $\Delta_{4} \neq 0$. The solution $\Delta_{4}=0$ implies $s_{i}=s_{i}^{0}$ which gives the infection-free steady state $\Pi_{0}\left(s_{1}^{0}, s_{2}^{0}, 0,0,0,0,0,0,0,0\right)$. The other solution $\Delta_{4} \neq 0$ admits 
a humoral-inactivated infection steady state $\Pi_{1}\left(\tilde{s}_{1}, \tilde{s}_{2}, \tilde{w}_{1}, \tilde{w}_{2}, \tilde{y}_{1}, \tilde{y}_{2}, \tilde{u}_{1}, \tilde{u}_{2}, \tilde{p}, 0\right)$ where the coordinates satisfy the equalities:

$$
\begin{aligned}
\pi_{i}\left(\tilde{s}_{i}\right) & =\lambda_{i} \chi_{i}\left(\tilde{s}_{i}, \tilde{p}\right), \\
\lambda_{1 i} e^{-\mu_{1 i} \tau_{1 i}} \chi_{i}\left(\tilde{s}_{i}, \tilde{p}\right) & =\left(\alpha_{i}+\beta_{i}\right) \psi_{1 i}\left(\tilde{w}_{i}\right), \\
\eta_{i} \psi_{2 i}\left(\tilde{y}_{i}\right) & =\lambda_{2 i} e^{-\mu_{2 i} \tau_{2 i}} \chi_{i}\left(\tilde{s}_{i}, \tilde{p}\right)+\alpha_{i} \psi_{1 i}\left(\tilde{w}_{i}\right), v_{i} \psi_{3 i}\left(\tilde{u}_{i}\right)=\lambda_{3 i} e^{-\mu_{3 i} \tau_{3 i}} \chi_{i}\left(\tilde{s}_{i}, \tilde{p}\right), \\
g \psi_{41}(\tilde{p}) & =\sum_{i=1}^{2}\left(N_{i} \eta_{i} e^{-\mu_{4 i} \tau_{4 i}} \psi_{2 i}\left(\tilde{y}_{i}\right)+M_{i} v_{i} e^{-\mu_{5 i} \tau_{5 i}} \psi_{3 i}\left(\tilde{u}_{i}\right)\right) .
\end{aligned}
$$

The other solution of Eq. (1.10) is $\psi_{41}(\bar{p})=\frac{\omega}{r}$, which yields $\bar{p}=\psi_{41}^{-1}\left(\frac{\omega}{r}\right)>0$. Substitute $p=\bar{p}$ in Eq. (1.5) and let $\Delta_{i}\left(s_{i}\right)=\pi_{i}\left(s_{i}\right)-\lambda_{i} \chi_{i}\left(s_{i}, \bar{p}\right)=0$. According to H1 and H2, $\Delta_{i}$ is strictly decreasing, $\Delta_{i}(0)=\pi_{i}(0)>0$ and $\Delta_{i}\left(s_{i}^{0}\right)=-\lambda_{i} \chi_{i}\left(s_{i}^{0}, \bar{p}\right)<0$. Thus, there exists a unique $\bar{s}_{i} \in\left(0, s_{i}^{0}\right)$ such that $\Delta_{\mathfrak{i}}\left(\bar{s}_{i}\right)=0$. It follows from Eqs. (1.9) and (1.12) that,

$$
\begin{aligned}
\bar{w}_{i} & =\Delta_{1 i}\left(\bar{s}_{i}\right)>0, \quad \bar{y}_{i}=\Delta_{2 i}\left(\bar{s}_{i}\right)>0, \quad \bar{u}_{i}=\Delta_{3 i}\left(\bar{s}_{i}\right)>0, \\
\bar{p} & =\psi_{41}^{-1}\left(\frac{\omega}{r}\right)>0, \bar{x}=\psi_{42}^{-1}\left(\frac{g}{\mu}\left(\sum_{i=1}^{2} \rho_{i} \frac{\chi_{i}\left(\bar{s}_{i}, \bar{p}\right)}{\psi_{41}(\bar{p})}-1\right)\right) .
\end{aligned}
$$

Thus, $\bar{x}>0$ when $\sum_{i=1}^{2} \rho_{i} \frac{x_{i}\left(\bar{s}_{i}, \bar{p}\right)}{\psi_{41}(\overline{\mathrm{p}})}>1$. Now we define the humoral immune response activation number as follows:

$$
\mathrm{R}_{1}=\sum_{i=1}^{2} \rho_{i} \frac{\chi_{i}\left(\bar{s}_{i}, \bar{p}\right)}{\psi_{41}(\bar{p})}
$$

If $R_{1}>1$, then $\bar{x}=\psi_{42}^{-1}\left(\frac{g}{\mu}\left(R_{1}-1\right)\right)>0$, and there exists a humoral-activated infection steady state $\Pi_{2}\left(\bar{s}_{1}, \bar{s}_{2}, \bar{w}_{1}, \bar{w}_{2}, \bar{y}_{1}, \bar{y}_{2}, \bar{u}_{1}, \bar{u}_{2}, \overline{\mathrm{p}}, \bar{x}\right)$. Clearly, from H2 and H4, we have

$$
R_{1}=\sum_{i=1}^{2} \rho_{i} \frac{\chi_{i}\left(\bar{s}_{i}, \bar{p}\right)}{\psi_{41}(\bar{p})} \leqslant \sum_{i=1}^{2} \lim _{p \rightarrow 0^{+}} \rho_{i} \frac{\chi_{i}\left(\bar{s}_{i}, p\right)}{\psi_{41}(\bar{p})} \leqslant \sum_{i=1}^{2} \frac{\rho_{i}}{\psi_{41}^{\prime}(0)} \frac{\partial \chi_{i}\left(\bar{s}_{i}, 0\right)}{\partial p} \leqslant \sum_{i=1}^{2} \frac{\rho_{i}}{\psi_{41}^{\prime}(0)} \frac{\partial \chi_{i}\left(s_{i}^{0}, 0\right)}{\partial p}=R_{0} .
$$

We will use the following equalities throughout the paper:

$$
\begin{aligned}
\ln \left(\frac{\chi_{i}\left(s_{i}\left(t-\tau_{1 i}\right), p\left(t-\tau_{1 i}\right)\right)}{\chi_{i}\left(s_{i}, p\right)}\right)= & \ln \left(\frac{\psi_{1 i}\left(\hat{w}_{i}\right) \chi_{i}\left(s_{i}\left(t-\tau_{1 i}\right), p\left(t-\tau_{1 i}\right)\right)}{\psi_{1 i}\left(w_{i}\right) \chi_{i}\left(\hat{s}_{i}, \hat{p}\right)}\right)+\ln \left(\frac{\chi_{i}\left(\hat{s}_{i}, \hat{p}\right)}{\chi_{i}\left(s_{i}, \hat{p}\right)}\right) \\
& +\ln \left(\frac{\psi_{41}(p) x_{i}\left(s_{i}, \hat{p}\right)}{\psi_{41}(\hat{p}) \chi_{i}\left(s_{i}, p\right)}\right)+\ln \left(\frac{\psi_{41}(\hat{p}) \psi_{2 i}\left(y_{i}\right)}{\psi_{41}(p) \psi_{2 i}\left(\hat{y}_{i}\right)}\right) \\
& +\ln \left(\frac{\psi_{2 i}(\hat{y}) \psi_{1 i}\left(w_{i}\right)}{\psi_{2 i}\left(y_{i}\right) \psi_{1 i}\left(\hat{w}_{i}\right)}\right), \\
\ln \left(\frac{\chi_{i}\left(s_{i}\left(t-\tau_{2 i}\right), p\left(t-\tau_{2 i}\right)\right)}{\chi_{i}\left(s_{i}, p\right)}\right)= & \ln \left(\frac{\psi_{2 i}\left(\hat{y}_{i}\right) \chi_{i}\left(s_{i}\left(t-\tau_{2 i}\right), p\left(t-\tau_{2 i}\right)\right)}{\psi_{2 i}\left(y_{i}\right) \chi_{i}\left(\hat{s}_{i}, \hat{p}\right)}\right)+\ln \left(\frac{\chi_{i}\left(\hat{s}_{i}, \hat{p}\right)}{\chi_{i}\left(s_{i}, \hat{p}\right)}\right) \\
& +\ln \left(\frac{\psi_{41}(\hat{p}) \psi_{2 i}\left(y_{i}\right)}{\psi_{41}(p) \psi_{2 i}\left(\hat{y}_{i}\right)}\right)+\ln \left(\frac{\psi_{41}(p) \chi_{i}\left(s_{i}, \hat{p}\right)}{\psi_{41}(\hat{p}) \chi_{i}\left(s_{i}, p\right)}\right), \\
\ln \left(\frac{\chi_{i}\left(s_{i}\left(t-\tau_{3 i}\right), p\left(t-\tau_{3 i}\right)\right)}{\chi_{i}\left(s_{i}, p\right)}\right)= & \ln \left(\frac{\psi_{3 i}\left(\hat{u}_{i}\right) \chi_{i}\left(s_{i}\left(t-\tau_{3 i}\right), p\left(t-\tau_{3 i}\right)\right)}{\psi_{3 i}\left(u_{i}\right) \chi_{i}\left(\hat{s}_{i}, \hat{p}\right)}\right)+\ln \left(\frac{\chi_{i}\left(\hat{s}_{i}, \hat{p}\right)}{\chi_{i}\left(s_{i}, \hat{p}\right)}\right) \\
& +\ln \left(\frac{\psi_{3 i}\left(u_{i}\right) \psi_{41}(\hat{p})}{\psi_{3 i}\left(\hat{u}_{i}\right) \psi_{41}(p)}\right)+\ln \left(\frac{\psi_{41}(p) \chi_{i}\left(s_{i}, \hat{p}\right)}{\psi_{41}(\hat{p}) \chi_{i}\left(s_{i}, p\right)}\right), \\
\ln \left(\frac{\psi_{2 i}\left(y_{i}\left(t-\tau_{4 i}\right)\right)}{\psi_{2 i}\left(y_{i}\right)}\right)= & \ln \left(\frac{\psi_{2 i}\left(y_{i}\left(t-\tau_{4 i}\right)\right) \psi_{41}(\hat{p})}{\psi_{2 i}\left(\hat{y}_{i}\right) \psi_{41}(p)}\right)+\ln \left(\frac{\psi_{2 i}\left(\hat{y}_{i}\right) \psi_{41}(p)}{\psi_{2 i}\left(y_{i}\right) \psi_{41}(\hat{p})}\right), \\
\ln \left(\frac{\psi_{3 i}\left(u_{i}\left(t-\tau_{5 i}\right)\right)}{\psi_{3 i}\left(u_{i}\right)}\right)= & \ln \left(\frac{\psi_{3 i}\left(u_{i}\left(t-\tau_{5 i}\right)\right) \psi_{41}(\hat{p})}{\psi_{3 i}\left(\hat{u}_{i}\right) \psi_{41}(p)}\right)+\ln \left(\frac{\psi_{3 i}\left(\hat{u}_{i}\right) \psi_{41}(p)}{\psi_{3 i}\left(u_{i}\right) \psi_{41}(\hat{p})}\right) .
\end{aligned}
$$




\section{Global properties}

The following theorems investigate the global stability of the steady states of system (1.3).

Let us denote $\left(s_{i}, w_{i}, y_{i}, u_{i}, p, x\right)=\left(s_{i}(t), w_{i}(t), y_{i}(t), u_{i}(t), p(t), x(t)\right)$.

Theorem 2.1. If $\mathrm{R}_{0} \leqslant 1$ and hypotheses $\mathrm{H} 1-\mathrm{H} 4$ are valid, then $\Pi_{0}$ is $G A S$.

Proof. Define a Lyapunov functional $v_{0}\left(s_{1}, s_{2}, w_{1}, w_{2}, y_{1}, y_{2}, u_{1}, u_{2}, p, x\right)$ as:

$$
\begin{aligned}
V_{0}= & \sum_{i=1}^{2} \rho_{i}\left[s_{i}-s_{i}^{0}-\int_{s_{i}^{0}}^{s_{i}} \lim _{p \rightarrow 0^{+}} \frac{\chi_{i}\left(s_{i}^{0}, p\right)}{\chi_{i}(\eta, p)} d \eta+\ell_{1 i} w_{i}+\ell_{2 i} y_{i}+\ell_{3 i} u_{i}\right. \\
& +\ell_{1 i} \lambda_{1 i} \int_{0}^{\tau_{1 i}} e^{-\mu_{1 i} \tau_{1 i}} \chi_{i}\left(s_{i}(t-\theta), p(t-\theta)\right) d \theta+\ell_{2 i} \lambda_{2 i} \int_{0}^{\tau_{2 i}} e^{-\mu_{2 i} \tau_{2 i}} \chi_{i}\left(s_{i}(t-\theta), p(t-\theta)\right) d \theta \\
& +\ell_{3 i} \lambda_{3 i} \int_{0}^{\tau_{3 i}} e^{-\mu_{3 i} \tau_{3 i}} \chi_{i}\left(s_{i}(t-\theta), p(t-\theta)\right) d \theta+\ell_{4 i} \eta_{i} \int_{0}^{\tau^{-\mu_{4 i}} \tau_{4 i}} \psi_{2 i}\left(y_{i}(t-\theta)\right) d \theta \\
& \left.+\ell_{5 i} v_{i} \int_{0}^{h_{5 i}} e^{-\mu_{5 i} \tau_{5 i}} \psi_{3 i}\left(u_{i}(t-\theta)\right) d \theta\right]+\ell_{61} p+\ell_{62} x,
\end{aligned}
$$

where $\ell_{1 i}, \ldots, \ell_{5 i}, \ell_{61}$, and $\ell_{62}$ satisfy the following equations:

$$
\begin{aligned}
& \lambda_{i}=\lambda_{1 i} \ell_{1 i} e^{-\mu_{1 i} \tau_{1 i}}+\lambda_{2 i} \ell_{2 i} e^{-\mu_{2 i} \tau_{2 i}}+\lambda_{3 i} \ell_{3 i} e^{-\mu_{3 i} \tau_{3 i}}, \quad\left(\alpha_{i}+\beta_{i}\right) \ell_{1 i}=\alpha_{i} \ell_{2 i}, \\
& \ell_{2 i}=\ell_{4 i} e^{-\mu_{4 i} \tau_{4 i}}, \ell_{3 i}=\ell_{5 i} e^{-\mu_{5 i} \tau_{5 i}}, \rho_{i} \ell_{4 i}=N_{i} \ell_{61}, \rho_{i} \ell_{5 i}=M_{i} \ell_{61}, \quad \mu \ell_{61}=r \ell_{62} .
\end{aligned}
$$

The solution of Eqs. (2.1) is given by

$$
\begin{aligned}
& \ell_{1 i}=\frac{\alpha_{i} N_{i} \lambda_{i} e^{-\mu_{4 i} \tau_{4 i}}}{\rho_{i} g\left(\alpha_{i}+\beta_{i}\right)}, \quad \ell_{2 i}=\frac{N_{i} \lambda_{i} e^{-\mu_{4 i} \tau_{4 i}}}{\rho_{i} g}, \quad \ell_{3 i}=\frac{M_{i} \lambda_{i} e^{-\mu_{5 i} \tau_{5 i}}}{\rho_{i} g}, \\
& \ell_{4 i}=\frac{N_{i} \lambda_{i}}{\rho_{i} g}, \quad \ell_{5 i}=\frac{M_{i} \lambda_{i}}{\rho_{i} g}, i=1,2, \quad \ell_{61}=\frac{\lambda_{i}}{g}, \quad \ell_{62}=\frac{\mu \lambda_{i}}{r g} .
\end{aligned}
$$

Clearly, $v_{0}\left(s_{1}, s_{2}, w_{1}, w_{2}, y_{1}, y_{2}, u_{1}, u_{2}, p, x\right)>0$ for all $s_{1}, s_{2}, w_{1}, w_{2}, y_{1}, y_{2}, u_{1}, u_{2}, p, x>0$ and $v_{0}\left(s_{1}^{0}, s_{2}^{0}, 0\right.$, $0,0,0,0,0,0,0)=0$. We calculate $\frac{d V_{0}}{d t}$ along the trajectories of (1.3) as:

$$
\begin{aligned}
\frac{d V_{0}}{d t}= & \sum_{i=1}^{2} \rho_{i}\left[\left(1-\lim _{p \rightarrow 0^{+}} \frac{\chi_{i}\left(s_{i}^{0}, p\right)}{\chi_{i}\left(s_{i}, p\right)}\right)\left(\pi_{i}\left(s_{i}\right)-\lambda_{i} x_{i}\left(s_{i}, p\right)\right)\right. \\
& +\ell_{1 i}\left(\lambda_{i 1} e^{-\mu_{1 i} \tau_{1 i}} \chi_{i}\left(s_{i}\left(t-\tau_{1 i}\right), p\left(t-\tau_{1 i}\right)\right)-\left(\alpha_{i}+\beta_{i}\right) \psi_{1 i}\left(w_{i}\right)\right) \\
& +\ell_{2 i}\left(\lambda_{2 i} e^{-\mu_{2 i} \tau_{2 i}} \chi_{i}\left(s_{i}\left(t-\tau_{2 i}\right), p\left(t-\tau_{2 i}\right)\right)+\alpha_{i} \psi_{1 i}\left(w_{i}\right)-\eta_{i} \psi_{2 i}\left(y_{i}\right)\right) \\
& +\ell_{3 i}\left(\lambda_{3 i} e^{-\mu_{3 i} \tau_{3 i}} \chi_{i}\left(s_{i}\left(t-\tau_{3 i}\right), p\left(t-\tau_{3 i}\right)\right)-v_{i} \psi_{3 i}\left(u_{i}\right)\right) \\
& +\ell_{1 i} \lambda_{1 i} e^{-\mu_{1 i} \tau_{1 i}}\left(\chi_{i}\left(s_{i}, p\right)-\chi_{i}\left(s_{i}\left(t-\tau_{1 i}\right), p\left(t-\tau_{1 i}\right)\right)\right) \\
& +\ell_{2 i} \lambda_{2 i} e^{-\mu_{2 i} \tau_{2 i}}\left(\chi_{i}\left(s_{i}, p\right)-\chi_{i}\left(s_{i}\left(t-\tau_{2 i}\right), p\left(t-\tau_{2 i}\right)\right)\right) \\
& +\ell_{3 i} \lambda_{3 i} e^{-\mu_{3 i} \tau_{3 i}}\left(\chi_{i}\left(s_{i}, p\right)-\chi_{i}\left(s_{i}\left(t-\tau_{3 i}\right), p\left(t-\tau_{3 i}\right)\right)\right) \\
& +\ell_{4 i} \eta_{i} e^{-\mu_{4 i} \tau_{4 i}}\left(\psi_{2 i}\left(y_{i}\right)-\psi_{2 i}\left(y_{i}\left(t-\tau_{4 i}\right)\right)\right) \\
& \left.+\ell_{5 i} v_{i} e^{-\mu_{5 i} \tau_{5 i}}\left(\psi_{3 i}\left(u_{i}\right)-\psi_{3 i}\left(u_{i}\left(t-\tau_{5 i}\right)\right)\right)\right] \\
& +\ell_{61} \sum_{i=1}^{2}\left(N_{i} \eta_{i} e^{-\mu_{4 i} \tau_{4 i}} \psi_{2 i}\left(y_{i}\left(t-\tau_{4 i}\right)\right)+M_{i} v_{i} e^{-\mu_{5 i} \tau_{5 i}} \psi_{3 i}\left(u_{i}\left(t-\tau_{5 i}\right)\right)\right) \\
& -\ell_{61} g \psi_{41}(p)-\ell_{61} \mu \psi_{41}(p) \psi_{42}(x)+\ell_{62}\left(r \psi_{41}(p) \psi_{42}(x)-\omega \psi_{42}(x)\right) .
\end{aligned}
$$


Collecting terms of Eq. (2.2) and using $\pi\left(s_{i}^{0}\right)=0$, we obtain

$$
\begin{aligned}
\frac{d V_{0}}{d t}= & \sum_{i=1}^{2} \rho_{i}\left(\pi_{i}\left(s_{i}\right)-\pi_{i}\left(s_{i}^{0}\right)\right)\left(1-\lim _{p \rightarrow 0^{+}} \frac{\chi_{i}\left(s_{i}^{0}, p\right)}{\chi_{i}\left(s_{i}, p\right)}\right) \\
& +\sum_{i=1}^{2} \rho_{i} \lambda_{i} \chi_{i}\left(s_{i}, p\right) \lim _{p \rightarrow 0^{+}} \frac{\chi_{i}\left(s_{i}^{0}, p\right)}{\chi_{i}\left(s_{i}, p\right)}-\ell_{61} g \psi_{41}(p)-\ell_{62} \omega \psi_{42}(x) \\
\leqslant & \sum_{i=1}^{2} \rho_{i}\left(\pi_{i}\left(s_{i}\right)-\pi_{i}\left(s_{i}^{0}\right)\right)\left(1-\lim _{p \rightarrow 0^{+}} \frac{\chi_{i}\left(s_{i}^{0}, p\right)}{\chi_{i}\left(s_{i}, p\right)}\right) \\
& +\left(\sum_{i=1}^{2} \rho_{i} \lambda_{i} \lim _{p \rightarrow 0^{+}} \frac{\chi_{i}\left(s_{i}, p\right)}{\psi_{41}(p)} \lim _{p \rightarrow 0^{+}} \frac{\chi_{i}\left(s_{i}^{0}, p\right)}{\chi_{i}\left(s_{i}, p\right)}-\ell_{61} g\right) \psi_{41}(p)-\ell_{62} \omega \psi_{42}(x) \\
= & \sum_{i=1}^{2} \rho_{i}\left(\pi_{i}\left(s_{i}\right)-\pi_{i}\left(s_{i}^{0}\right)\right)\left(1-\frac{\partial \chi_{i}\left(s_{i}^{0}, 0\right) / \partial p}{\partial \chi_{i}\left(s_{i}, 0\right) / \partial p}\right) \\
& +\ell_{61} g\left(\sum_{i=1}^{2} \frac{\rho_{i} \lambda_{i}}{\ell_{61} g \psi_{41}^{\prime}(0)} \frac{\partial x_{i}\left(s_{i}^{0}, 0\right)}{\partial p}-1\right) \psi_{41}(p)-\ell_{62} \omega \psi_{42}(x) \\
= & \sum_{i=1}^{2} \rho_{i}\left(\pi_{i}\left(s_{i}\right)-\pi_{i}\left(s_{i}^{0}\right)\right)\left(1-\frac{\partial \chi_{i}\left(s_{i}^{0}, 0\right) / \partial p}{\partial \chi_{i}\left(s_{i}, 0\right) / \partial p}\right)+\ell_{61} g\left(R_{0}-1\right) \psi_{41}(p)-\ell_{62} \omega \psi_{42}(x) .
\end{aligned}
$$

By $\mathrm{H} 1$ and $\mathrm{H} 2$, we obtain

$$
\left(\pi_{\mathfrak{i}}\left(s_{i}\right)-\pi_{\mathfrak{i}}\left(s_{i}^{0}\right)\right)\left(1-\frac{\partial \chi_{i}\left(s_{i}^{0}, 0\right) / \partial p}{\partial \chi_{i}\left(s_{i}, 0\right) / \partial p}\right) \leqslant 0 .
$$

Therefore, if $R_{0} \leqslant 1$, then $\frac{d V_{0}}{d t} \leqslant 0$ for $s_{i}, p, x \in(0, \infty)$. Clearly, $\frac{d V_{0}}{d t}=0$ at $\Pi_{0}$. Applying LIP, we get that $\Pi_{0}$ is GAS.

Lemma 2.2. If $\mathrm{R}_{0}>1$ and hypotheses $\mathrm{H} 1-\mathrm{H} 4$ are valid, then

$$
\operatorname{sgn}\left(R_{1}-1\right)=\operatorname{sgn}(\tilde{p}-\bar{p})=\operatorname{sgn}\left(\bar{s}_{i}-\tilde{s}_{i}\right) .
$$

Proof. Using hypotheses $\mathrm{H} 1$ and $\mathrm{H} 2$, for $\tilde{s}_{i}, \bar{s}_{i}, \tilde{\mathrm{p}}, \overline{\mathrm{p}}>0$, we get

$$
\begin{aligned}
\left(\tilde{s}_{i}-\bar{s}_{i}\right)\left(\pi_{i}\left(\bar{s}_{i}\right)-\pi_{i}\left(\tilde{s}_{i}\right)\right) & >0, \\
\left(\bar{s}_{i}-\tilde{s}_{i}\right)\left(\chi_{i}\left(\bar{s}_{i}, \bar{p}\right)-\chi_{i}\left(\tilde{s}_{i}, \bar{p}\right)\right) & >0, \\
(\bar{p}-\tilde{p})\left(\chi_{i}\left(\tilde{s}_{i}, \bar{p}\right)-\chi_{i}\left(\tilde{s}_{i}, \tilde{p}\right)\right) & >0,
\end{aligned}
$$

and from hypothesis $\mathrm{H} 4$, we obtain

$$
(\tilde{p}-\bar{p})\left(\frac{\chi_{i}\left(\tilde{s}_{i}, \bar{p}\right)}{\psi_{41}(\bar{p})}-\frac{\chi_{i}\left(\tilde{s}_{i}, \tilde{p}\right)}{\psi_{41}(\tilde{p})}\right)>0 .
$$

First, we show that $\operatorname{sgn}(\tilde{p}-\bar{p})=\operatorname{sgn}\left(\bar{s}_{i}-\tilde{s}_{i}\right)$. Suppose that $\operatorname{sgn}(\bar{p}-\tilde{p})=\operatorname{sgn}\left(\bar{s}_{i}-\tilde{s}_{i}\right)$. Using the steady state conditions of $\Pi_{1}$ and $\Pi_{2}$, we obtain

$$
\pi_{i}\left(\bar{s}_{i}\right)-\pi_{i}\left(\tilde{s}_{i}\right)=\lambda_{i}\left[\chi_{i}\left(\bar{s}_{i}, \bar{p}\right)-\chi_{i}\left(\tilde{s}_{i}, \tilde{p}\right)\right]=\lambda_{i}\left[\left(\chi_{i}\left(\bar{s}_{i}, \bar{p}\right)-\chi_{i}\left(\tilde{s}_{i}, \bar{p}\right)\right)+\left(\chi_{i}\left(\tilde{s}_{i}, \bar{p}\right)-\chi_{i}\left(\tilde{s}_{i}, \tilde{p}\right)\right)\right] .
$$

Therefore, from the inequalities (2.3)-(2.5) we obtain sgn $\left(\bar{s}_{i}-\tilde{s}_{i}\right)=\operatorname{sgn}\left(\tilde{s}_{i}-\bar{s}_{i}\right)$, which is a contradiction; hence, $\operatorname{sgn}(\tilde{p}-\bar{p})=\operatorname{sgn}\left(\bar{s}_{i}-\tilde{s}_{i}\right)$. Using Eqs. (1.14) and the definition of $R_{1}$, we get

$$
R_{1}-1=\sum_{i=1}^{2} \rho_{i}\left(\frac{\chi_{i}\left(\bar{s}_{i}, \bar{p}\right)}{\psi_{41}(\bar{p})}-\frac{\chi_{i}\left(\tilde{s}_{i}, \tilde{p}\right)}{\psi_{41}(\tilde{p})}\right)=\sum_{i=1}^{2} \rho_{i}\left[\frac{1}{\psi_{41}(\bar{p})}\left(\chi_{i}\left(\bar{s}_{i}, \bar{p}\right)-\chi_{i}\left(\tilde{s}_{i}, \bar{p}\right)\right)+\frac{\chi_{i}\left(\tilde{s}_{i}, \bar{p}\right)}{\psi_{41}(\bar{p})}-\frac{\chi_{i}\left(\tilde{s}_{i}, \tilde{p}\right)}{\psi_{41}(\tilde{p})}\right] .
$$

Thus, from inequalities (2.4) and (2.6) we obtain $\operatorname{sgn}\left(R_{1}-1\right)=\operatorname{sgn}(\tilde{p}-\bar{p})$.

Theorem 2.3. Suppose that hypotheses $\mathrm{H} 1-\mathrm{H} 4$ are valid, $\Pi_{1}$ exists, and $\mathrm{R}_{1} \leqslant 1$, then $\Pi_{1}$ is $G A S$. 
Proof. Let $\mathrm{v}_{1}\left(\mathrm{~s}_{1}, \mathrm{~s}_{2}, w_{1}, w_{2}, \mathrm{y}_{1}, \mathrm{y}_{2}, \mathrm{u}_{1}, \mathrm{u}_{2}, \mathrm{p}, \mathrm{x}\right)$ as

$$
\begin{aligned}
& V_{1}=\sum_{i=1}^{2} \rho_{i}\left[s_{i}-\tilde{s}_{i}-\int_{\tilde{s}_{i}}^{s_{i}} \frac{\chi_{i}\left(\tilde{s}_{i}, \tilde{p}\right)}{\chi_{i}(\eta, \tilde{p})} d \eta+\ell_{1 i}\left(w_{i}-\tilde{w}_{i}-\int_{\tilde{w}_{i}}^{w_{i}} \frac{\psi_{1 i}\left(\tilde{w}_{i}\right)}{\psi_{1 i}(\eta)} d \eta\right)\right. \\
& +\ell_{2 i}\left(y_{i}-\tilde{y}_{i}-\int_{\tilde{y}_{i}}^{y_{i}} \frac{\psi_{2 i}\left(\tilde{y}_{i}\right)}{\psi_{2 i}(\eta)} d \eta\right)+\ell_{3 i}\left(u_{i}-\tilde{u}_{i}-\int_{\tilde{u}_{i}}^{u_{i}} \frac{\psi_{3 i}\left(\tilde{u}_{i}\right)}{\psi_{3 i}(\eta)} d \eta\right) \\
& +\ell_{1 i} \lambda_{1 i} \chi_{i}\left(\tilde{s}_{i}, \tilde{p}\right) \int_{0}^{\tau_{1 i}} e^{-\mu_{1 i} \tau_{1 i} F}\left(\frac{\chi_{i}\left(s_{i}(t-\theta), p(t-\theta)\right)}{\chi_{i}\left(\tilde{s}_{i}, \tilde{p}\right)}\right) d \theta \\
& +\ell_{2 i} \lambda_{2 i} \chi_{i}\left(\tilde{s}_{i}, \tilde{p}\right) \int_{0}^{\tau_{2 i}} e^{-\mu_{2 i} \tau_{2 i}} F\left(\frac{\chi_{i}\left(s_{i}(t-\theta), p(t-\theta)\right)}{\chi_{i}\left(\tilde{s}_{i}, \tilde{p}\right)}\right) d \theta \\
& +\ell_{3 i} \lambda_{3 i} \chi_{i}\left(\tilde{s}_{i}, \tilde{p}\right) \int_{0}^{\tau_{3 i}} e^{-\mu_{3 i} \tau_{3 i}} F\left(\frac{\chi_{i}\left(s_{i}(t-\theta), p(t-\theta)\right)}{\chi_{i}\left(\tilde{s}_{i}, \tilde{p}\right)}\right) d \theta \\
& +\ell_{4 i} \eta_{i} \psi_{2 i}\left(\tilde{y}_{i}\right) \int_{0}^{\tau_{4 i}} e^{-\mu_{4 i} \tau_{4 i} F}\left(\frac{\psi_{2 i}\left(y_{i}(t-\theta)\right)}{\psi_{2 i}\left(\tilde{y}_{i}\right)}\right) d \theta \\
& \left.+\ell_{5 i} v_{i} \psi_{2 i}\left(\tilde{u}_{i}\right) \int_{0}^{\tau_{5 i}} e^{-\mu_{5 i} \tau_{5 i} F}\left(\frac{\psi_{3 i}\left(u_{i}(t-\theta)\right)}{\psi_{3 i}\left(\tilde{u}_{i}\right)}\right) d \theta\right]+\ell_{61}\left(p-\tilde{p}-\int_{\tilde{p}}^{p} \frac{\psi_{41}(\tilde{p})}{\psi_{41}(\eta)} d \eta\right)+\ell_{62} x .
\end{aligned}
$$

We can see that, $v_{1}\left(s_{1}, s_{2}, w_{1}, w_{2}, y_{1}, y_{2}, u_{1}, u_{2}, p, x\right)>0$ for all $s_{1}, s_{2}, w_{1}, w_{2}, y_{1}, y_{2}, u_{1}, u_{2}, p, x>0$ and $\mathrm{V}_{1}\left(\tilde{s}_{1}, \tilde{s}_{2}, \tilde{w}_{1}, \tilde{w}_{2}, \tilde{y}_{1}, \tilde{y}_{2}, \tilde{u}_{1}, \tilde{u}_{2}, \tilde{p}, 0\right)=0$. Calculating $\frac{d V_{1}}{d t}$ along the solutions of (1.3) we obtain:

$$
\begin{aligned}
\frac{d v_{1}}{d t}= & \sum_{i=1}^{2} \rho_{i}\left[\left(1-\frac{\chi_{i}\left(\tilde{s}_{i}, \tilde{p}\right)}{\chi_{i}\left(s_{i}, \tilde{p}\right)}\right)\left(\pi_{i}\left(s_{i}\right)-\lambda_{i} x_{i}\left(s_{i}, p\right)\right)\right. \\
& +\ell_{1 i}\left(1-\frac{\psi_{1 i}\left(\tilde{w}_{i}\right)}{\psi_{1 i}\left(w_{i}\right)}\right)\left(\lambda_{1 i} e^{-\mu_{1 i} \tau_{1 i}} \chi_{i}\left(s_{i}\left(t-\tau_{1 i}\right), p\left(t-\tau_{1 i}\right)\right)-\left(\alpha_{i}+\beta_{i}\right) \psi_{1 i}\left(w_{i}\right)\right) \\
& +\ell_{2 i}\left(1-\frac{\psi_{2 i}\left(\tilde{y}_{i}\right)}{\psi_{2 i}\left(y_{i}\right)}\right)\left(\lambda_{2 i} e^{-\mu_{2 i} \tau_{2 i}} \chi_{i}\left(s_{i}\left(t-\tau_{2 i}\right), p\left(t-\tau_{2 i}\right)\right)+\alpha_{i} \psi_{1 i}\left(w_{i}\right)-\eta_{i} \psi_{2 i}\left(y_{i}\right)\right) \\
& +\ell_{3 i}\left(1-\frac{\psi_{3 i}\left(\tilde{u}_{i}\right)}{\psi_{3 i}\left(u_{i}\right)}\right)\left(\lambda_{3 i} e^{-\mu_{3 i} \tau_{3 i}} \chi_{i}\left(s_{i}\left(t-\tau_{3 i}\right), p\left(t-\tau_{3 i}\right)\right)-v_{i} \psi_{3 i}\left(u_{i}\right)\right) \\
& +\ell_{1 i} \lambda_{1 i} e^{-\mu_{1 i} \tau_{1 i}}\left(\chi_{i}\left(s_{i}, p\right)-\chi_{i}\left(s_{i}\left(t-\tau_{1 i}\right), p\left(t-\tau_{1 i}\right)\right)\right) \\
& +\chi_{i}\left(\tilde{s}_{i}, \tilde{p}\right) \ell_{1 i} \lambda_{1 i} e^{-\mu_{1 i} \tau_{1 i}} \ln \left(\frac{\chi_{i}\left(s_{i}\left(t-\tau_{1 i}\right), p\left(t-\tau_{1 i}\right)\right)}{\chi_{i}\left(s_{i}, p\right)}\right) \\
& +\ell_{2 i} \lambda_{2 i} e^{-\mu_{2 i} \tau_{2 i}}\left(\chi_{i}\left(s_{i}, p\right)-\chi_{i}\left(s_{i}\left(t-\tau_{2 i}\right), p\left(t-\tau_{2 i}\right)\right)\right) \\
& +\chi_{i}\left(\tilde{s}_{i}, \tilde{p}\right) \ell_{2 i} \lambda_{2 i} e^{-\mu_{2 i} \tau_{2 i}} \ln \left(\frac{\chi_{i}\left(s_{i}\left(t-\tau_{2 i}\right), p\left(t-\tau_{2 i}\right)\right)}{\chi_{i}\left(s_{i}, p\right)}\right) \\
& +\ell_{3 i} \lambda_{3 i} e^{-\mu_{3 i} \tau_{3 i}}\left(\chi_{i}\left(s_{i}, p\right)-\chi_{i}\left(s_{i}\left(t-\tau_{3 i}\right), p\left(t-\tau_{3 i}\right)\right)\right) \\
& +\chi_{i}\left(\tilde{s}_{i}, \tilde{p}\right) \ell_{3 i} \lambda_{3 i} e^{-\mu_{3 i} \tau_{3 i}} \ln \left(\frac{\chi_{i}\left(s_{i}\left(t-\tau_{3 i}\right), p\left(t-\tau_{3 i}\right)\right)}{\chi_{i}\left(s_{i}, p\right)}\right) \\
& +\ell_{4 i} \eta_{i} e^{-\mu_{4 i} \tau_{4 i}}\left(\psi_{2 i}\left(y_{i}\right)-\psi_{2 i}\left(y_{i}\left(t-\tau_{4 i}\right)\right)+\psi_{2 i}\left(\tilde{y}_{i}\right) \ln \left(\frac{\psi_{2 i}\left(y_{i}\left(t-\tau_{4 i}\right)\right)}{\psi_{2 i}\left(y_{i}\right)}\right)\right) \\
& \left.+\ell_{5 i} v_{i} e^{-\mu_{5 i} \tau_{5 i}}\left(\psi_{3 i}\left(u_{i}\right)-\psi_{3 i}\left(u_{i}\left(t-\tau_{5 i}\right)\right)+\psi_{3 i}\left(\tilde{u}_{i}\right) \ln \left(\frac{\psi_{3 i}\left(u_{i}\left(t-\tau_{5 i}\right)\right)}{\psi_{3 i}\left(u_{i}\right)}\right)\right)\right]
\end{aligned}
$$




$$
\begin{aligned}
& +\ell_{61}\left(1-\frac{\psi_{41}(\tilde{p})}{\psi_{41}(p)}\right) \sum_{i=1}^{2}\left(N_{i} \eta_{i} e^{-\mu_{4 i} \tau_{4 i}} \psi_{2 i}\left(y_{i}\left(t-\tau_{4 i}\right)\right)+M_{i} v_{i} e^{-\mu_{5 i} \tau_{5 i}} \psi_{3 i}\left(u_{i}\left(t-\tau_{5 i}\right)\right)\right) \\
& -\ell_{61}\left(1-\frac{\psi_{41}(\tilde{p})}{\psi_{41}(p)}\right)\left(g \psi_{41}(p)+\mu \psi_{41}(p) \psi_{42}(x)\right)+\ell_{62}\left(r \psi_{41}(p) \psi_{42}(x)-\omega \psi_{42}(x)\right) .
\end{aligned}
$$

Collecting terms of Eq. (2.7) and applying $\pi_{i}\left(\tilde{s}_{i}\right)=\lambda_{i} \chi_{i}\left(\tilde{s}_{i}, \tilde{p}\right)$ we get

$$
\begin{aligned}
& \frac{d V_{1}}{d t}=\sum_{i=1}^{2} \rho_{i}\left[\left(\pi_{i}\left(s_{i}\right)-\pi_{i}\left(\tilde{s}_{i}\right)\right)\left(1-\frac{\chi_{i}\left(\tilde{s}_{i}, \tilde{p}\right)}{\chi_{i}\left(s_{i}, \tilde{p}\right)}\right)+\lambda_{i} \chi_{i}\left(\tilde{s}_{i}, \tilde{p}\right)\left(1-\frac{\chi_{i}\left(\tilde{s}_{i}, \tilde{p}\right)}{\chi_{i}\left(s_{i}, \tilde{p}\right)}\right)+\lambda_{i} \chi_{i}\left(s_{i}, p\right) \frac{\chi_{i}\left(\tilde{s}_{i}, \tilde{p}\right)}{\chi_{i}\left(s_{i}, \tilde{p}\right)}\right. \\
& -\ell_{1 i} \lambda_{1 i} e^{-\mu_{1 i} \tau_{1 i}} \frac{\chi_{i}\left(s_{i}\left(t-\tau_{1 i}\right), p\left(t-\tau_{1 i}\right)\right) \psi_{1 i}\left(\tilde{w}_{i}\right)}{\psi_{1 i}\left(w_{i}\right)}+\ell_{1 i}\left(\alpha_{i}+\beta_{i}\right) \psi_{1 i}\left(\tilde{w}_{i}\right) \\
& +\ell_{2 i} \eta_{i} \psi_{2 i}\left(\tilde{y}_{i}\right)-\ell_{2 i} \lambda_{2 i} e^{-\mu_{2 i} \tau_{2 i}} \frac{\chi_{i}\left(s_{i}\left(t-\tau_{2 i}\right), p\left(t-\tau_{2 i}\right)\right) \psi_{2 i}\left(\tilde{y}_{i}\right)}{\psi_{2 i}\left(y_{i}\right)} \\
& -\alpha_{i} \ell_{2 i} \frac{\psi_{1 i}\left(w_{i}\right) \psi_{2 i}\left(\tilde{y}_{i}\right)}{\psi_{2 i}\left(y_{i}\right)}-\ell_{3 i} \lambda_{3 i} e^{-\mu_{3 i} \tau_{3 i}} \frac{\chi_{i}\left(s_{i}\left(t-\tau_{3 i}\right), p\left(t-\tau_{3 i}\right)\right) \psi_{3 i}\left(\tilde{u}_{i}\right)}{\psi_{3 i}\left(u_{i}\right)} \\
& +\ell_{3 i} v_{i} \psi_{3 i}\left(\tilde{u}_{i}\right)+\ell_{1 i} \lambda_{1 i} \chi_{i}\left(\tilde{s}_{i}, \tilde{p}\right) e^{-\mu_{1 i} \tau_{1 i}} \ln \left(\frac{\chi_{i}\left(s_{i}\left(t-\tau_{1 i}\right), p\left(t-\tau_{1 i}\right)\right)}{\chi_{i}\left(s_{i}, p\right)}\right) \\
& +\ell_{2 i} \lambda_{2 i} \chi_{i}\left(\tilde{s}_{i}, \tilde{p}\right) e^{-\mu_{2 i} \tau_{2 i}} \ln \left(\frac{\chi_{i}\left(s_{i}\left(t-\tau_{2 i}\right), p\left(t-\tau_{2 i}\right)\right)}{\chi_{i}\left(s_{i}, p\right)}\right) \\
& +\ell_{3 i} \lambda_{3 i} \chi_{i}\left(\tilde{s}_{i}, \tilde{p}\right) e^{-\mu_{3 i} \tau_{3 i}} \ln \left(\frac{\chi_{i}\left(s_{i}\left(t-\tau_{3 i}\right), p\left(t-\tau_{3 i}\right)\right)}{\chi_{i}(x, p)}\right) \\
& +\ell_{4 i} \eta_{i} \psi_{2 i}\left(\tilde{y}_{i}\right) e^{-\mu_{4 i} \tau_{4 i}} \ln \left(\frac{\psi_{2 i}\left(y_{i}\left(t-\tau_{4 i}\right)\right)}{\psi_{2 i}\left(y_{i}\right)}\right) \\
& \left.+\ell_{5 i} v_{i} \psi_{3 i}\left(\tilde{u}_{i}\right) e^{-\mu_{5 i} \tau_{5 i}} \ln \left(\frac{\psi_{3 i}\left(u_{i}\left(t-\tau_{5 i}\right)\right)}{\psi_{3 i}\left(u_{i}\right)}\right)\right]-\ell_{61} g \psi_{41}(p) \\
& -\ell_{61} \sum_{i=1}^{2} N_{i} \eta_{i} e^{-\mu_{4 i} \tau_{4 i}} \frac{\psi_{2 i}\left(y_{i}\left(t-\tau_{4 i}\right)\right) \psi_{41}(\tilde{p})}{\psi_{41}(p)} \\
& -\ell_{61} \sum_{i=1}^{2} M_{i} v_{i} e^{-\mu_{5 i}} \tau_{5 i} \frac{\psi_{3 i}\left(u_{i}\left(t-\tau_{5 i}\right)\right) \psi_{41}(\tilde{p})}{\psi_{41}(p)}+\ell_{61} g \psi_{41}(\tilde{p})+\mu \ell_{61} \psi_{41}(\tilde{p}) \psi_{42}(x)-\ell_{62} \omega \psi_{42}(x) \text {. }
\end{aligned}
$$

From the conditions of the steady state $\Pi_{1}$ :

$$
\begin{aligned}
\left(\alpha_{i}+\beta_{i}\right) \psi_{1 i}\left(\tilde{w}_{i}\right) & =\lambda_{1 i} e^{-\mu_{1 i} \tau_{1 i}} \chi_{i}\left(\tilde{s}_{i}, \tilde{p}\right), & \ell_{2 i} \eta_{i} \psi_{2 i}\left(\tilde{y}_{i}\right) & =\left(\ell_{1 i} \lambda_{1 i} e^{-\mu_{1 i} \tau_{1 i}}+\ell_{2 i} \lambda_{2 i} e^{-\mu_{2 i} \tau_{2 i}}\right) \chi_{i}\left(\tilde{s}_{i}, \tilde{p}\right), \\
v_{i} \psi_{3 i}\left(\tilde{u}_{i}\right) & =\lambda_{3 i} e^{-\mu_{3 i} \tau_{3 i}} \chi_{i}\left(\tilde{s}_{i}, \tilde{p}\right), & \ell_{61} g \psi_{41}(\tilde{p}) & =\sum_{i=1}^{2} \rho_{i} \lambda_{i} \chi_{i}\left(\tilde{s}_{i}, \tilde{p}\right),
\end{aligned}
$$

we get

$$
\begin{aligned}
\frac{\mathrm{d} V_{1}}{\mathrm{dt}}= & \sum_{i=1}^{2} \rho_{i}\left[\left(\pi_{i}\left(s_{i}\right)-\pi_{i}\left(\tilde{s}_{i}\right)\right)\left(1-\frac{\chi_{i}\left(\tilde{s}_{i}, \tilde{p}\right)}{\chi_{i}\left(s_{i}, \tilde{p}\right)}\right)+\lambda_{i} \chi_{i}\left(\tilde{s}_{i}, \tilde{p}\right)\left(1-\frac{\chi_{i}\left(\tilde{s}_{i}, \tilde{p}\right)}{\chi_{i}\left(s_{i}, \tilde{p}\right)}\right)\right. \\
& +\ell_{1 i} \lambda_{1 i} e^{-\mu_{1 i} \tau_{1 i}} \chi_{i}\left(\tilde{s}_{i}, \tilde{p}\right)+\lambda_{i} \chi_{i}\left(\tilde{s}_{i}, \tilde{p}\right)\left(\frac{\chi_{i}\left(s_{i}, p\right)}{\left.\chi_{i}\left(s_{i}, \tilde{p}\right)\right)}-\frac{\psi_{41}(\mathfrak{p})}{\psi_{41}(\tilde{p})}\right) \\
& -\ell_{1 i} \lambda_{1 i} \chi_{i}\left(\tilde{s}_{i}, \tilde{p}\right) e^{-\mu_{1 i} \tau_{1 i}} \frac{\chi_{i}\left(s_{i}\left(t-\tau_{1 i}\right), p\left(t-\tau_{1 i}\right)\right) \psi_{1 i}\left(\tilde{w}_{i}\right)}{\chi_{i}\left(\tilde{s}_{i}, \tilde{p}\right) \psi_{1 i}\left(w_{i}\right)} \\
& -\ell_{2 i} \lambda_{2 i} \chi_{i}\left(\tilde{s}_{i}, \tilde{p}\right) e^{-\mu_{2 i} \tau_{2 i}} \frac{\chi_{i}\left(s_{i}\left(t-\tau_{2 i}\right), p\left(t-\tau_{2 i}\right)\right) \psi_{2 i}\left(\tilde{y}_{i}\right)}{\chi_{i}\left(\tilde{s}_{i}, \tilde{p}\right) \psi_{2 i}\left(y_{i}\right)} \\
& -\ell_{1 i} \lambda_{1 i} e^{-\mu_{1 i} \tau_{1 i}} \chi_{i}\left(\tilde{s}_{i}, \tilde{p}\right) \frac{\psi_{2 i}\left(\tilde{y}_{i}\right) \psi_{1 i}\left(w_{i}\right)}{\psi_{2 i}\left(y_{i}\right) \psi_{1 i}\left(\tilde{w}_{i}\right)}+\left(\ell_{1 i} \lambda_{1 i} e^{-\mu_{1 i} \tau_{1 i}}+\ell_{2 i} \lambda_{2 i} e^{-\mu_{2 i} \tau_{2 i}}\right) \chi_{i}\left(\tilde{s}_{i}, \tilde{p}\right)
\end{aligned}
$$




$$
\begin{aligned}
& +\ell_{3 i} \lambda_{3 i} e^{-\mu_{3 i} \tau_{3 i}} \chi_{i}\left(\tilde{s}_{i}, \tilde{p}\right)-\ell_{3 i} \lambda_{3 i} \chi_{i}\left(\tilde{s}_{i}, \tilde{p}\right) e^{-\mu_{3 i} \tau_{3 i}} \frac{\chi_{i}\left(s_{i}\left(t-\tau_{3 i}\right), p\left(t-\tau_{3 i}\right)\right) \psi_{3 i}\left(\tilde{u}_{i}\right)}{\chi_{i}\left(\tilde{s}_{i}, \tilde{p}\right) \psi_{3 i}\left(u_{i}\right)} \\
& +\ell_{1 i} \lambda_{1 i} \chi_{i}\left(\tilde{s}_{i}, \tilde{p}\right) e^{-\mu_{1 i} \tau_{1 i}} \ln \left(\frac{\chi_{i}\left(s_{i}\left(t-\tau_{1 i}\right), p\left(t-\tau_{1 i}\right)\right)}{\chi_{i}\left(s_{i}, p\right)}\right) \\
& +\ell_{2 i} \lambda_{2 i} \chi_{i}\left(\tilde{s}_{i}, \tilde{p}\right) e^{-\mu_{2 i} \tau_{2 i}} \ln \left(\frac{\chi_{i}\left(s_{i}\left(t-\tau_{2 i}\right), p\left(t-\tau_{2 i}\right)\right)}{\chi_{i}\left(s_{i}, p\right)}\right) \\
& +\ell_{3 i} \lambda_{3 i} \chi_{i}\left(\tilde{s}_{i}, \tilde{p}\right) e^{-\mu_{3 i} \tau_{3 i}} \ln \left(\frac{\chi_{i}\left(s_{i}\left(t-\tau_{3 i}\right), p\left(t-\tau_{3 i}\right)\right)}{\chi_{i}\left(s_{i}, p\right)}\right) \\
& +\left(\ell_{1 i} \lambda_{1 i} e^{-\mu_{1 i} \tau_{1 i}}+\ell_{2 i} \lambda_{2 i} e^{-\mu_{2 i} \tau_{2 i}}\right) \chi_{i}\left(\tilde{s}_{i}, \tilde{p}\right) \ln \left(\frac{\psi_{2 i}\left(y_{i}\left(t-\tau_{4 i}\right)\right)}{\psi_{2 i}\left(y_{i}\right)}\right) \\
& \left.+\ell_{3 i} \lambda_{3 i} e^{-\mu_{3 i} \tau_{3 i}} \chi_{i}\left(\tilde{s}_{i}, \tilde{p}\right) \ln \left(\frac{\psi_{3 i}\left(u_{i}\left(t-\tau_{5 i}\right)\right)}{\psi_{3 i}\left(u_{i}\right)}\right)\right]+\sum_{i=1}^{2} \rho_{i} \lambda_{i} \chi_{i}\left(\tilde{s}_{i}, \tilde{p}\right) \\
& -\sum_{i=1}^{2} \rho_{i}\left(\ell_{1 i} \lambda_{1 i} e^{-\mu_{1 i} \tau_{1 i}}+\ell_{2 i} \lambda_{2 i} e^{-\mu_{2 i} \tau_{2 i}}\right) \chi_{i}\left(\tilde{s}_{i}, \tilde{p}\right) \frac{\psi_{2 i}\left(y_{i}\left(t-\tau_{4 i}\right)\right) \psi_{41}(\tilde{p})}{\psi_{2 i}(\tilde{y} i) \psi_{41}(p)} \\
& -\sum_{i=1}^{2} \rho_{i} \ell_{3 i} \lambda_{3 i} e^{-\mu_{3 i} \tau_{3 i}} \chi_{i}\left(\tilde{s}_{i}, \tilde{p}\right) \frac{\psi_{3 i}\left(u_{i}\left(t-\tau_{5 i}\right)\right) \psi_{41}(\tilde{p})}{\psi_{3 i}\left(\tilde{u}_{i}\right) \psi_{41}(p)}+r \ell_{62}\left(\psi_{41}(\tilde{p})-\frac{\omega}{r}\right) \psi_{42}(x) .
\end{aligned}
$$

Using the equalities (1.15) with $\hat{s}_{i}=\tilde{s}_{i}, \hat{w}_{i}=\tilde{w}_{i}, \hat{y}_{i}=\tilde{y}_{i}, \hat{u}_{i}=\tilde{u}_{i}$ and $\hat{p}=\tilde{p}$, we can obtain

$$
\begin{aligned}
& \frac{d V_{1}}{d t}=\sum_{i=1}^{2} \rho_{i}\left[\left(\pi_{i}\left(s_{i}\right)-\pi_{i}\left(\tilde{s}_{i}\right)\right)\left(1-\frac{\chi_{i}\left(\tilde{s}_{i}, \tilde{p}\right)}{\chi_{i}\left(s_{i}, \tilde{p}\right)}\right)\right. \\
& +\lambda_{i} \chi_{i}\left(\tilde{s}_{i}, \tilde{p}\right)\left(\frac{\chi_{i}\left(s_{i}, p\right)}{\chi_{i}\left(s_{i}, \tilde{p}\right)}-\frac{\psi_{41}(p)}{\psi_{41}(\tilde{p})}-1+\frac{\psi_{41}(p) \chi_{i}\left(s_{i}, \tilde{p}\right)}{\psi_{41}(\tilde{p}) \chi_{i}\left(s_{i}, p\right)}\right) \\
& -\lambda_{i} \chi_{i}\left(\tilde{s}_{i}, \tilde{p}\right)\left(\frac{\chi_{i}\left(\tilde{s}_{i}, \tilde{p}\right)}{\chi_{i}\left(s_{i}, \tilde{p}\right)}-1-\ln \left(\frac{\chi_{i}\left(\tilde{s}_{i}, \tilde{p}\right)}{\chi_{i}\left(s_{i}, \tilde{p}\right)}\right)\right) \\
& -\lambda_{i} \chi_{i}\left(\tilde{s}_{i}, \tilde{p}\right)\left(\frac{\psi_{41}(p) \chi_{i}\left(s_{i}, \tilde{p}\right)}{\psi_{41}(\tilde{p}) \chi_{i}\left(s_{i}, p\right)}-1-\ln \left(\frac{\psi_{41}(p) \chi_{i}\left(s_{i}, \tilde{p}\right)}{\psi_{41}(\tilde{p}) \chi_{i}\left(s_{i}, p\right)}\right)\right) \\
& -\ell_{1 i} \lambda_{1 i} \chi_{i}\left(\tilde{s}_{i}, \tilde{p}\right) e^{-\mu_{1 i} \tau_{1 i}}\left(\frac{\psi_{1 i}\left(\tilde{w}_{i}\right) \chi_{i}\left(s_{i}\left(t-\tau_{1 i}\right), p\left(t-\tau_{1 i}\right)\right)}{\psi_{1 i}\left(w_{i}\right) \chi_{i}\left(\tilde{s}_{i}, \tilde{p}\right)}\right. \\
& \left.-1-\ln \left(\frac{\psi_{1 i}\left(\tilde{w}_{i}\right) \chi_{i}\left(s_{i}\left(t-\tau_{1 i}\right), p\left(t-\tau_{1 i}\right)\right)}{\psi_{1 i}\left(w_{i}\right) \chi_{i}\left(\tilde{s}_{i}, \tilde{p}\right)}\right)\right) \\
& -\ell_{2 i} \lambda_{2 i} \chi_{i}\left(\tilde{s}_{i}, \tilde{p}\right) e^{-\mu_{2 i} \tau_{2 i}}\left(\frac{\psi_{2 i}\left(\tilde{y}_{i}\right) \chi_{i}\left(s_{i}\left(t-\tau_{2 i}\right), p\left(t-\tau_{2 i}\right)\right)}{\psi_{2 i}\left(y_{i}\right) \chi_{i}\left(\tilde{s}_{i}, \tilde{p}\right)}\right. \\
& \left.-1-\ln \left(\frac{\psi_{2 i}\left(\tilde{y}_{i}\right) \chi_{i}\left(s_{i}\left(t-\tau_{2 i}\right), p\left(t-\tau_{2 i}\right)\right)}{\psi_{2 i}\left(y_{i}\right) \chi_{i}\left(\tilde{s}_{i}, \tilde{p}\right)}\right)\right) \\
& -\ell_{3 i} \lambda_{3 i} \chi_{i}\left(\tilde{s}_{i}, \tilde{p}\right) e^{-\mu_{3 i} \tau_{3 i}}\left(\frac{\psi_{3 i}\left(\tilde{u}_{i}\right) \chi_{i}\left(s_{i}\left(t-\tau_{3 i}\right), p\left(t-\tau_{3 i}\right)\right)}{\psi_{3 i}\left(u_{i}\right) \chi_{i}\left(\tilde{s}_{i}, \tilde{p}\right)}\right. \\
& \left.-1-\ln \left(\frac{\psi_{3 i}\left(\tilde{u}_{i}\right) \chi_{i}\left(s_{i}\left(t-\tau_{3 i}\right), p\left(t-\tau_{3 i}\right)\right)}{\psi_{3 i}\left(u_{i}\right) \chi_{i}\left(\tilde{s}_{i}, \tilde{p}\right)}\right)\right) \\
& -\ell_{1 i} \lambda_{1 i} e^{-\mu_{1 i} \tau_{1 i}} \chi_{i}\left(\tilde{s}_{i}, \tilde{p}\right)\left(\frac{\psi_{2 i}\left(\tilde{y}_{i}\right) \psi_{1 i}\left(w_{i}\right)}{\psi_{2 i}\left(y_{i}\right) \psi_{1 i}\left(\tilde{w}_{i}\right)}-1-\ln \left(\frac{\psi_{2 i}\left(\tilde{y}_{i}\right) \psi_{1 i}\left(w_{i}\right)}{\psi_{2 i}\left(y_{i}\right) \psi_{1 i}\left(\tilde{w}_{i}\right)}\right)\right) \\
& -\left(\ell_{1 i} \lambda_{1 i} e^{-\mu_{1 i} \tau_{1 i}}+\ell_{2 i} \lambda_{2 i} e^{-\mu_{2 i} \tau_{2 i}}\right) \chi_{i}\left(\tilde{s}_{i}, \tilde{p}\right)\left(\frac{\psi_{2 i}\left(y_{i}\left(t-\tau_{4 i}\right)\right) \psi_{41}(\tilde{p})}{\psi_{2 i}\left(\tilde{y}_{i}\right) \psi_{41}(p)}\right. \\
& \left.-1-\ln \left(\frac{\psi_{2 i}\left(y_{i}\left(t-\tau_{4 i}\right)\right) \psi_{41}(\tilde{p})}{\psi_{2 i}\left(\tilde{y}_{i}\right) \psi_{41}(p)}\right)\right)-\ell_{3 i} \lambda_{3 i} e^{-\mu_{3 i} \tau_{3 i}} \chi_{i}\left(\tilde{s}_{i}, \tilde{p}\right)\left(\frac{\psi_{3 i}\left(u_{i}\left(t-\tau_{5 i}\right)\right) \psi_{41}(\tilde{p})}{\psi_{3 i}\left(\tilde{u}_{i}\right) \psi_{41}(p)}-1\right. \\
& \left.\left.-\ln \left(\frac{\psi_{3 i}\left(u_{i}\left(t-\tau_{5 i}\right)\right) \psi_{41}(\tilde{p})}{\psi_{3 i}\left(\tilde{u}_{i}\right) \psi_{41}(p)}\right)\right)\right]+r l_{62}\left(\psi_{41}(\tilde{p})-\psi_{41}(\bar{p})\right) \psi_{42}(x) .
\end{aligned}
$$


Eq. (2.8) becomes:

$$
\begin{aligned}
& \frac{d V_{1}}{d t}=\sum_{i=1}^{2} \rho_{i}\left[\left(\pi_{i}\left(s_{i}\right)-\pi_{i}\left(\tilde{s}_{i}\right)\right)\left(1-\frac{\chi_{i}\left(\tilde{s}_{i}, \tilde{p}\right)}{\chi_{i}\left(s_{i}, \tilde{p}\right)}\right)+\lambda_{i} \chi_{i}\left(\tilde{s}_{i}, \tilde{p}\right)\left(\frac{\chi_{i}\left(s_{i}, p\right)}{\chi_{i}\left(s_{i}, \tilde{p}\right)}-\frac{\psi_{41}(p)}{\psi_{41}(\tilde{p})}\right)\left(1-\frac{\chi_{i}\left(s_{i}, \tilde{p}\right)}{\chi_{i}\left(s_{i}, p\right)}\right)\right. \\
& -\lambda_{i} \chi_{i}\left(\tilde{s}_{i}, \tilde{p}\right)\left(F\left(\frac{\chi_{i}\left(\tilde{s}_{i}, \tilde{p}\right)}{\chi_{i}\left(s_{i}, \tilde{p}\right)}\right)+F\left(\frac{\psi_{41}(p) \chi_{i}\left(s_{i}, \tilde{p}\right)}{\psi_{41}(\tilde{p}) \chi_{i}\left(s_{i}, p\right)}\right)\right)-\ell_{1 i} \lambda_{1 i} e^{-\mu_{1 i} \tau_{1 i}} \chi_{i}\left(\tilde{s}_{i}, \tilde{p}\right) F\left(\frac{\psi_{2 i}\left(\tilde{y}_{i}\right) \psi_{1 i}\left(w_{i}\right)}{\psi_{2 i}\left(y_{i}\right) \psi_{1 i}\left(\tilde{w}_{i}\right)}\right) \\
& -\ell_{1 i} \lambda_{1 i} \chi_{i}\left(\tilde{s}_{i}, \tilde{p}\right) e^{-\mu_{1 i} \tau_{1 i}} F\left(\frac{\psi_{1 i}\left(\tilde{w}_{i}\right) \chi_{i}\left(s_{i}\left(t-\tau_{1 i}\right), p\left(t-\tau_{1 i}\right)\right)}{\psi_{1 i}\left(w_{i}\right) \chi_{i}\left(\tilde{s}_{i}, \tilde{p}\right)}\right) \\
& -\ell_{2 i} \lambda_{2 i} \chi_{i}\left(\tilde{s}_{i}, \tilde{p}\right) e^{-\mu_{2 i} \tau_{2 i}} F\left(\frac{\psi_{2 i}\left(\tilde{y}_{i}\right) \chi_{i}\left(s_{i}\left(t-\tau_{2 i}\right), p\left(t-\tau_{2 i}\right)\right)}{\psi_{2 i}\left(y_{i}\right) \chi_{i}\left(\tilde{s}_{i}, \tilde{p}\right)}\right) \\
& -\ell_{3 i} \lambda_{3 i} \chi_{i}\left(\tilde{s}_{i}, \tilde{p}\right) e^{-\mu_{3 i} \tau_{3 i}} F\left(\frac{\psi_{3 i}\left(\tilde{u}_{i}\right) \chi_{i}\left(s_{i}\left(t-\tau_{3 i}\right), p\left(t-\tau_{3 i}\right)\right)}{\psi_{3 i}\left(u_{i}\right) \chi_{i}\left(\tilde{s}_{i}, \tilde{p}\right)}\right) \\
& -\left(\ell_{1 i} \lambda_{1 i} e^{-\mu_{1 i} \tau_{1 i}}+\ell_{2 i} \lambda_{2 i} e^{-\mu_{2 i} \tau_{2 i}}\right) \chi_{i}\left(\tilde{s}_{i}, \tilde{p}\right) F\left(\frac{\psi_{2 i}\left(y_{i}\left(t-\tau_{4 i}\right)\right) \psi_{41}(\tilde{p})}{\psi_{2 i}\left(\tilde{y}_{i}\right) \psi_{41}(p)}\right) \\
& \left.-\ell_{3 i} \lambda_{3 i} e^{-\mu_{3 i} \tau_{3 i}} \chi_{i}\left(\tilde{s}_{i}, \tilde{p}\right) F\left(\frac{\psi_{3 i}\left(u_{i}\left(t-\tau_{5 i}\right)\right) \psi_{41}(\tilde{p})}{\psi_{3 i}\left(\tilde{u}_{i}\right) \psi_{41}(p)}\right)\right]+r \ell_{62}\left(\psi_{41}(\tilde{p})-\psi_{41}(\bar{p})\right) \psi_{42}(x) .
\end{aligned}
$$

$\mathrm{H} 1, \mathrm{H} 2, \mathrm{H} 4$, Remark 1.1, Lemma 2.2, and the condition $\mathrm{R}_{1} \leqslant 1$ imply that

$$
\left(\pi_{i}\left(s_{i}\right)-\pi_{i}\left(\tilde{s}_{i}\right)\right)\left(1-\frac{\chi_{i}\left(\tilde{s}_{i}, \tilde{p}\right)}{\chi_{i}\left(s_{i}, \tilde{p}\right)}\right) \leqslant 0, \quad\left(\frac{\chi_{i}\left(s_{i}, p\right)}{\chi_{i}\left(s_{i}, \tilde{p}\right)}-\frac{\psi_{41}(p)}{\psi_{41}(\tilde{p})}\right)\left(1-\frac{\chi_{i}\left(s_{i}, \tilde{p}\right)}{\chi_{i}\left(s_{i}, p\right)}\right) \leqslant 0, \quad \psi_{41}(\tilde{p})-\psi_{41}(\bar{p}) \leqslant 0 .
$$

It follows that, for all $s_{i}, y_{i}, p, x>0$, we have $\frac{d V_{1}}{d t} \leqslant 0$ and $\frac{d V_{1}}{d t}=0$ at $\Pi_{1}$. By LIP, $\Pi_{1}$ is GAS.

Theorem 2.4. If $\mathrm{R}_{1}>1$ and hypotheses $\mathrm{H} 1-\mathrm{H} 4$ are valid, then $\Pi_{2}$ is $G A S$.

Proof. Define $v_{2}\left(s_{1}, s_{2}, w_{1}, w_{2}, y_{1}, y_{2}, u_{1}, u_{2}, p, x\right)$ as

$$
\begin{aligned}
& v_{2}=\sum_{i=1}^{2} \rho_{i}\left[s_{i}-\bar{s}_{i}-\int_{\bar{s}_{i}}^{s_{i}} \frac{\chi_{i}\left(\bar{s}_{i}, \bar{p}\right)}{\chi_{i}(\eta, \bar{p})} d \eta+\ell_{1 i}\left(w_{i}-\bar{w}_{i}-\int_{\bar{w}_{i}}^{w_{i}} \frac{\psi_{1 i}\left(\bar{w}_{i}\right)}{\psi_{1 i}(\eta)} d \eta\right)\right. \\
& +\ell_{2 i}\left(y_{i}-\bar{y}_{i}-\int_{\bar{y}_{i}}^{y_{i}} \frac{\psi_{2 i}\left(\bar{y}_{i}\right)}{\psi_{2 i}(\eta)} d \eta\right)+\ell_{3 i}\left(u_{i}-\bar{u}_{i}-\int_{\bar{u}_{i}}^{u_{i}} \frac{\psi_{3 i}\left(\bar{u}_{i}\right)}{\psi_{3 i}(\eta)} d \eta\right) \\
& +\ell_{1 i} \lambda_{1 i} \chi_{i}\left(\bar{s}_{i}, \bar{p}\right) \int_{0}^{\tau_{1 i}} e^{-\mu_{1 i} \tau_{1 i}} F\left(\frac{\chi_{i}\left(s_{i}(t-\theta), p(t-\theta)\right)}{\chi_{i}\left(\bar{s}_{i}, \bar{p}\right)}\right) d \theta \\
& +\ell_{2 i} \lambda_{2 i} \chi_{i}\left(\bar{s}_{i}, \bar{p}\right) \int_{0}^{\tau_{2 i}} e^{-\mu_{2 i} \tau_{2 i}} F\left(\frac{\chi_{i}\left(s_{i}(t-\theta), p(t-\theta)\right)}{\chi_{i}\left(\bar{s}_{i}, \bar{p}\right)}\right) d \theta \\
& +\ell_{3 i} \lambda_{3 i} \chi_{i}\left(\bar{s}_{i}, \bar{p}\right) \int_{0}^{\tau_{3 i}} e^{-\mu_{3 i} \tau_{3 i}} F\left(\frac{\chi_{i}\left(s_{i}(t-\theta), p(t-\theta)\right)}{\chi_{i}\left(\bar{s}_{i}, \bar{p}\right)}\right) d \theta \\
& \left.+\ell_{4 i} \eta_{i} \psi_{2 i}\left(\bar{y}_{i}\right) \int_{0}^{\tau_{4 i}} e^{-\mu_{4 i} \tau_{4 i}} F\left(\frac{\psi_{2 i}\left(y_{i}(t-\theta)\right)}{\psi_{2 i}\left(\bar{y}_{i}\right)}\right) d \theta+\ell_{5 i} \nu_{i} \psi_{3 i}\left(\bar{u}_{i}\right) \int_{0}^{\tau_{5 i}} e^{-\mu_{5 i} \tau_{5 i} F}\left(\frac{\psi_{3 i}\left(u_{i}(t-\theta)\right)}{\psi_{3 i}\left(\bar{u}_{i}\right)}\right) d \theta\right] \\
& +\ell_{61}\left(p-\bar{p}-\int_{\bar{p}}^{p} \frac{\psi_{41}(\bar{p})}{\psi_{41}(\eta)} d \eta\right)+\ell_{62}\left(x-\bar{x}-\int_{\bar{x}}^{x} \frac{\psi_{42}(\bar{x})}{\psi_{42}(\eta)} d \eta\right) .
\end{aligned}
$$


Note that, $v_{2}\left(s_{1}, s_{2}, w_{1}, w_{2}, y_{1}, y_{2}, u_{1}, u_{2}, p, x\right)>0$ for all $s_{1}, s_{2}, w_{1}, w_{2}, y_{1}, y_{2}, p, x>0$ and $v_{2}\left(\bar{s}_{1}, \bar{s}_{2}, \bar{w}_{1}, \bar{w}_{2}, \bar{y}_{1}\right.$, $\left.\bar{y}_{2}, \bar{u}_{1}, \bar{u}_{2}, \bar{p}, \bar{x}\right)=0$. Calculating $\frac{d V_{2}}{d t}$ along the solutions of model (1.3), we get

$$
\begin{aligned}
& \frac{d V_{2}}{d t}=\sum_{i=1}^{2} \rho_{i}\left[\left(1-\frac{\chi_{i}\left(\bar{s}_{i}, \bar{p}\right)}{\chi_{i}\left(s_{i}, \bar{p}\right)}\right)\left(\pi_{i}\left(s_{i}\right)-\lambda_{i} \chi_{i}\left(s_{i}, p\right)\right)\right. \\
& +\ell_{1 i}\left(1-\frac{\psi_{1 i}\left(\bar{w}_{i}\right)}{\psi_{1 i}\left(w_{i}\right)}\right)\left(\lambda_{1 i} e^{-\mu_{1 i} \tau_{1 i}} \chi_{i}\left(s_{i}\left(t-\tau_{1 i}\right), p\left(t-\tau_{1 i}\right)\right)-\left(\alpha_{i}+\beta_{i}\right) \psi_{1 i}\left(w_{i}\right)\right) \\
& +\ell_{2 i}\left(1-\frac{\psi_{2 i}\left(\bar{y}_{i}\right)}{\psi_{2 i}\left(y_{i}\right)}\right)\left(\lambda_{2 i} e^{-\mu_{2 i} \tau_{2 i}} \chi_{i}\left(s_{i}\left(t-\tau_{2 i}\right), p\left(t-\tau_{2 i}\right)\right)+\alpha_{i} \psi_{1 i}\left(w_{i}\right)-\eta_{i} \psi_{2 i}\left(y_{i}\right)\right) \\
& +\ell_{3 i}\left(1-\frac{\psi_{3 i}\left(\bar{u}_{i}\right)}{\psi_{3 i}\left(u_{i}\right)}\right)\left(\lambda_{3 i} e^{-\mu_{3 i} \tau_{3 i}} \chi_{i}\left(s_{i}\left(t-\tau_{3 i}\right), p\left(t-\tau_{3 i}\right)\right)-v_{i} \psi_{3 i}\left(u_{i}\right)\right) \\
& +\ell_{1 i} \lambda_{1 i} e^{-\mu_{1 i} \tau_{1 i}}\left(\chi_{i}\left(s_{i}, p\right)-\chi_{i}\left(s_{i}\left(t-\tau_{1 i}\right), p\left(t-\tau_{1 i}\right)\right)\right) \\
& +\chi_{i}\left(\bar{s}_{i}, \bar{p}\right) \ell_{1 i} \lambda_{1 i} e^{-\mu_{1 i} \tau_{1 i}} \ln \left(\frac{\chi_{i}\left(s_{i}\left(t-\tau_{1 i}\right), p\left(t-\tau_{1 i}\right)\right)}{\chi_{i}\left(s_{i}, p\right)}\right) \\
& +\ell_{2 i} \lambda_{2 i} e^{-\mu_{2 i} \tau_{2 i}}\left(\chi_{i}\left(s_{i}, p\right)-\chi_{i}\left(s_{i}\left(t-\tau_{2 i}\right), p\left(t-\tau_{2 i}\right)\right)\right) \\
& +\chi_{i}\left(\bar{s}_{i}, \bar{p}\right) \ell_{2 i} \lambda_{2 i} e^{-\mu_{2 i} \tau_{2 i}} \ln \left(\frac{\chi_{i}\left(s_{i}\left(t-\tau_{2 i}\right), p\left(t-\tau_{2 i}\right)\right)}{\chi_{i}\left(s_{i}, p\right)}\right) \\
& +\ell_{3 i} \lambda_{3 i} e^{-\mu_{3 i} \tau_{3 i}}\left(\chi_{i}\left(s_{i}, p\right)-\chi_{i}\left(s_{i}\left(t-\tau_{3 i}\right), p\left(t-\tau_{3 i}\right)\right)\right) \\
& +\chi_{i}\left(\bar{s}_{i}, \bar{p}\right) \ell_{3 i} \lambda_{3 i} e^{-\mu_{3 i} \tau_{3 i}} \ln \left(\frac{\chi_{i}\left(s_{i}\left(t-\tau_{3 i}\right), p\left(t-\tau_{3 i}\right)\right)}{\chi_{i}\left(s_{i}, p\right)}\right) \\
& +\ell_{4 i} \eta_{i} e^{-\mu_{4 i} \tau_{4 i}}\left(\psi_{2 i}\left(y_{i}\right)-\psi_{2 i}\left(y_{i}\left(t-\tau_{4 i}\right)\right)+\psi_{2 i}\left(\bar{y}_{i}\right) \ln \left(\frac{\psi_{2 i}\left(y_{i}\left(t-\tau_{4 i}\right)\right)}{\psi_{2 i}\left(y_{i}\right)}\right)\right) \\
& \left.+\ell_{5 i} v_{i} e^{-\mu_{5 i} \tau_{5 i}}\left(\psi_{3 i}\left(u_{i}\right)-\psi_{3 i}\left(u_{i}\left(t-\tau_{5 i}\right)\right)+\psi_{3 i}\left(\bar{u}_{i}\right) \ln \left(\frac{\psi_{3 i}\left(u_{i}\left(t-\tau_{5 i}\right)\right)}{\psi_{3 i}\left(u_{i}\right)}\right)\right)\right] \\
& +\ell_{61}\left(1-\frac{\psi_{41}(\bar{p})}{\psi_{41}(p)}\right) \sum_{i=1}^{2}\left(N_{i} \eta_{i} e^{-\mu_{4 i} \tau_{4 i}} \psi_{2 i}\left(y_{i}\left(t-\tau_{4 i}\right)\right)+M_{i} v_{i} e^{-\mu_{5 i} \tau_{5 i}} \psi_{3 i}\left(u_{i}\left(t-\tau_{5 i}\right)\right)\right) \\
& -\ell_{61}\left(1-\frac{\psi_{41}(\bar{p})}{\psi_{41}(p)}\right)\left(g \psi_{41}(p)+\mu \psi_{41}(p) \psi_{42}(x)\right) \\
& +\ell_{62}\left(1-\frac{\psi_{42}(\bar{x})}{\psi_{42}(x)}\right)\left(r \psi_{41}(p) \psi_{42}(x)-\omega \psi_{42}(x)\right) \text {. }
\end{aligned}
$$

Collecting terms of Eq. (2.9) and applying $\pi_{i}\left(\bar{s}_{i}\right)=\lambda_{i} \chi_{i}\left(\bar{s}_{i}, \bar{p}\right)$ we get

$$
\begin{aligned}
\frac{d V_{2}}{d t}= & \sum_{i=1}^{2} \rho_{i}\left[\left(\pi_{i}\left(s_{i}\right)-\pi_{i}\left(\bar{s}_{i}\right)\right)\left(1-\frac{\chi_{i}\left(\bar{s}_{i}, \bar{p}\right)}{\chi_{i}\left(s_{i}, \bar{p}\right)}\right)+\lambda_{i} \chi_{i}\left(\bar{s}_{i}, \bar{p}\right)\left(1-\frac{\chi_{i}\left(\bar{s}_{i}, \bar{p}\right)}{\chi_{i}\left(s_{i}, \bar{p}\right)}\right)+\lambda_{i} \chi_{i}\left(s_{i}, p\right) \frac{\chi_{i}\left(\bar{s}_{i}, \bar{p}\right)}{\chi_{i}\left(s_{i}, \bar{p}\right)}\right. \\
& -\ell_{1 i} \lambda_{1 i} e^{-\mu_{1 i} \tau_{1 i}} \frac{\chi_{i}\left(s_{i}\left(t-\tau_{1 i}\right), p\left(t-\tau_{1 i}\right)\right) \psi_{1 i}\left(\bar{w}_{i}\right)}{\psi_{1 i}\left(w_{i}\right)}+\ell_{1 i}\left(\alpha_{i}+\beta_{i}\right) \psi_{1 i}\left(\bar{w}_{i}\right) \\
& +\ell_{2 i} \eta_{i} \psi_{2 i}\left(\bar{y}_{i}\right)-\ell_{2 i} \lambda_{2 i} e^{-\mu_{2 i} \tau_{2 i}} \frac{\chi_{i}\left(s_{i}\left(t-\tau_{2 i}\right), p\left(t-\tau_{2 i}\right)\right) \psi_{2 i}\left(\bar{y}_{i}\right)}{\psi_{2 i}\left(y_{i}\right)} \\
& -\alpha_{i} \ell_{2 i} \frac{\psi_{1 i}\left(w_{i}\right) \psi_{2 i}\left(\bar{y}_{i}\right)}{\psi_{2 i}\left(y_{i}\right)}-\ell_{3 i} \lambda_{3 i} e^{-\mu_{3 i} \tau_{3 i}} \frac{\chi_{i}\left(s_{i}\left(t-\tau_{3 i}\right), p\left(t-\tau_{3 i}\right)\right) \psi_{3 i}\left(\bar{u}_{i}\right)}{\psi_{3 i}\left(u_{i}\right)} \\
& +\ell_{3 i} v_{i} \psi_{3 i}\left(\bar{u}_{i}\right)+\ell_{1 i} \lambda_{1 i} \chi_{i}\left(\bar{s}_{i}, \bar{p}\right) e^{-\mu_{1 i} \tau_{1 i}} \ln \left(\frac{\chi_{i}\left(s_{i}\left(t-\tau_{1 i}\right), p\left(t-\tau_{1 i}\right)\right)}{\chi_{i}\left(s_{i}, p\right)}\right) \\
& +\ell_{2 i} \lambda_{2 i} \chi_{i}\left(\bar{s}_{i}, \bar{p}\right) e^{-\mu_{2 i} \tau_{2 i}} \ln \left(\frac{\chi_{i}\left(s_{i}\left(t-\tau_{2 i}\right), p\left(t-\tau_{2 i}\right)\right)}{\chi_{i}\left(s_{i}, p\right)}\right)
\end{aligned}
$$




$$
\begin{aligned}
& +\ell_{3 i} \lambda_{3 i} \chi_{i}\left(\bar{s}_{i}, \bar{p}\right) e^{-\mu_{3 i} \tau_{3 i}} \ln \left(\frac{\chi_{i}\left(s_{i}\left(t-\tau_{3 i}\right), p\left(t-\tau_{3 i}\right)\right)}{\chi_{i}(x, p)}\right) \\
& +\ell_{4 i} \eta_{i} \psi_{2 i}\left(\bar{y}_{i}\right) e^{-\mu_{4 i} \tau_{4 i}} \ln \left(\frac{\psi_{2 i}\left(y_{i}\left(t-\tau_{4 i}\right)\right)}{\psi_{2 i}\left(y_{i}\right)}\right) \\
& \left.+\ell_{5 i} v_{i} \psi_{3 i}\left(\bar{u}_{i}\right) e^{-\mu_{5 i} \tau_{5 i}} \ln \left(\frac{\psi_{3 i}\left(u_{i}\left(t-\tau_{5 i}\right)\right)}{\psi_{3 i}\left(u_{i}\right)}\right)\right]-\ell_{61} g \psi_{41}(p) \\
& +\ell_{61} g \psi_{41}(\bar{p})-\ell_{61} \sum_{i=1}^{2} N_{i} \eta_{i} e^{-\mu_{4 i} \tau_{4 i}} \frac{\psi_{2 i}\left(y_{i}\left(t-\tau_{4 i}\right)\right) \psi_{41}(\bar{p})}{\psi_{41}(p)} \\
& -\ell_{61} \sum_{i=1}^{2} M_{i} v_{i} e^{-\mu_{5 i} \tau_{5 i}} \frac{\psi_{3 i}\left(u_{i}\left(t-\tau_{5 i}\right)\right) \psi_{41}(\bar{p})}{\psi_{41}(p)}+\mu \ell_{61} \psi_{41}(\bar{p}) \psi_{42}(x) \\
& -\ell_{62} \omega \psi_{42}(x)+\ell_{62} \omega \psi_{42}(\bar{x})-r \ell_{62} \psi_{41}(p) \psi_{42}(\bar{x}) .
\end{aligned}
$$

Using the steady state conditions for $\Pi_{2}$ :

$$
\begin{aligned}
& \left(\alpha_{i}+\beta_{i}\right) \psi_{1 i}\left(\bar{w}_{i}\right)=\lambda_{1 i} e^{-\mu_{1 i} \tau_{1 i}} \chi_{i}\left(\bar{s}_{i}, \bar{p}\right), \quad \ell_{2 i} \eta_{i} \psi_{2 i}\left(\bar{y}_{i}\right)=\left(\ell_{1 i} \lambda_{1 i} e^{-\mu_{1 i} \tau_{1 i}}+\ell_{2 i} \lambda_{2 i} e^{-\mu_{2 i} \tau_{2 i}}\right) \chi_{i}\left(\bar{s}_{i}, \bar{p}\right), \\
& v_{i} \psi_{3 i}\left(\bar{u}_{i}\right)=\lambda_{3 i} e^{-\mu_{3 i} \tau_{3 i}} \chi_{i}\left(\bar{s}_{i}, \bar{p}\right), \quad \ell_{61} g \psi_{41}(\bar{p})=\sum_{i=1}^{2} \rho_{i} \lambda_{i} \chi_{i}\left(\bar{s}_{i}, \bar{p}\right)-\mu \ell_{61} \psi_{41}(\bar{p}) \psi_{42}(\bar{x}), \\
& \ell_{61} g \psi_{41}(p)=\frac{\psi_{41}(p)}{\psi_{41}(\bar{p})} \sum_{i=1}^{2} \rho_{i} \lambda_{i} \chi_{i}\left(\bar{s}_{i}, \bar{p}\right)-\mu \ell_{61} \psi_{41}(p) \psi_{42}(\bar{x}),
\end{aligned}
$$

we obtain

$$
\begin{aligned}
& \frac{\mathrm{d} V_{2}}{\mathrm{dt}}=\sum_{i=1}^{2} \rho_{i}\left[\left(\pi_{i}\left(s_{i}\right)-\pi_{i}\left(\bar{s}_{i}\right)\right)\left(1-\frac{\chi_{i}\left(\bar{s}_{i}, \bar{p}\right)}{\chi_{i}\left(s_{i}, \bar{p}\right)}\right)\right. \\
& +\lambda_{i} \chi_{i}\left(\bar{s}_{i}, \bar{p}\right)\left(1-\frac{\chi_{i}\left(\bar{s}_{i}, \bar{p}\right)}{\chi_{i}\left(s_{i}, \bar{p}\right)}\right)+\lambda_{i} \chi_{i}\left(\bar{s}_{i}, \bar{p}\right)\left(\frac{\chi_{i}\left(s_{i}, p\right)}{\left.\chi_{i}\left(s_{i}, \bar{p}\right)\right)}-\frac{\psi_{41}(p)}{\psi_{41}(\bar{p})}\right) \\
& +\ell_{1 i} \lambda_{1 i} e^{-\mu_{1 i} \tau_{1 i}} \chi_{i}\left(\bar{s}_{i}, \bar{p}\right)-\ell_{1 i} \lambda_{1 i} \chi_{i}\left(\bar{s}_{i}, \bar{p}\right) e^{-\mu_{1 i} \tau_{1 i}} \frac{\chi_{i}\left(s_{i}\left(t-\tau_{1 i}\right), p\left(t-\tau_{1 i}\right)\right) \psi_{1 i}\left(\bar{w}_{i}\right)}{\chi_{i}\left(\bar{s}_{i}, \bar{p}\right) \psi_{1 i}\left(w_{i}\right)} \\
& -\ell_{2 i} \lambda_{2 i} \chi_{i}\left(\bar{s}_{i}, \bar{p}\right) e^{-\mu_{2 i} \tau_{2 i}} \frac{\chi_{i}\left(s_{i}\left(t-\tau_{2 i}\right), p\left(t-\tau_{2 i}\right)\right) \psi_{2 i}\left(\bar{y}_{i}\right)}{\chi_{i}\left(\bar{s}_{i}, \bar{p}\right) \psi_{2 i}\left(y_{i}\right)} \\
& -\ell_{1 i} \lambda_{1 i} e^{-\mu_{1 i} \tau_{1 i}} \chi_{i}\left(\bar{s}_{i}, \bar{p}\right) \frac{\psi_{2 i}\left(\bar{y}_{i}\right) \psi_{1 i}\left(w_{i}\right)}{\psi_{2 i}\left(y_{i}\right) \psi_{1 i}\left(\bar{w}_{i}\right)}+\left(\ell_{1 i} \lambda_{1 i} e^{-\mu_{1 i} \tau_{1 i}}+\ell_{2 i} \lambda_{2 i} e^{-\mu_{2 i} \tau_{2 i}}\right) \chi_{i}\left(\bar{s}_{i}, \bar{p}\right) \\
& +\ell_{3 i} \lambda_{3 i} e^{-\mu_{3 i} \tau_{3 i}} \chi_{i}\left(\bar{s}_{i}, \bar{p}\right)-\ell_{3 i} \lambda_{3 i} \chi_{i}\left(\bar{s}_{i}, \bar{p}\right) e^{-\mu_{3 i}} \tau_{3 i} \frac{\chi_{i}\left(s_{i}\left(t-\tau_{3 i}\right), p\left(t-\tau_{3 i}\right) \psi_{3 i}\left(\bar{u}_{i}\right)\right)}{\chi_{i}\left(\bar{s}_{i}, \bar{p}\right) \psi_{3 i}\left(u_{i}\right)} \\
& +\ell_{1 i} \lambda_{1 i} \chi_{i}\left(\bar{s}_{i}, \bar{p}\right) e^{-\mu_{1 i} \tau_{1 i}} \ln \left(\frac{\chi_{i}\left(s_{i}\left(t-\tau_{1 i}\right), p\left(t-\tau_{1 i}\right)\right)}{\chi_{i}\left(s_{i}, p\right)}\right) \\
& +\ell_{2 i} \lambda_{2 i} \chi_{i}\left(\bar{s}_{i}, \bar{p}\right) e^{-\mu_{2 i} \tau_{2 i}} \ln \left(\frac{\chi_{i}\left(s_{i}\left(t-\tau_{2 i}\right), p\left(t-\tau_{2 i}\right)\right)}{\chi_{i}\left(s_{i}, p\right)}\right) \\
& +\ell_{3 i} \lambda_{3 i} \chi_{i}\left(\bar{s}_{i}, \bar{p}\right) e^{-\mu_{3 i} \tau_{3 i}} \ln \left(\frac{\chi_{i}\left(s_{i}\left(t-\tau_{3 i}\right), p\left(t-\tau_{3 i}\right)\right)}{\chi_{i}\left(s_{i}, p\right)}\right) \\
& +\left(\ell_{1 i} \lambda_{1 i} e^{-\mu_{1 i} \tau_{1 i}}+\ell_{2 i} \lambda_{2 i} e^{-\mu_{2 i} \tau_{2 i}}\right) \chi_{i}\left(\bar{s}_{i}, \bar{p}\right) \ln \left(\frac{\psi_{2 i}\left(y_{i}\left(t-\tau_{4 i}\right)\right)}{\psi_{2 i}\left(y_{i}\right)}\right) \\
& \left.+\ell_{3 i} \lambda_{3 i} e^{-\mu_{3 i} \tau_{3 i}} \chi_{i}\left(\bar{s}_{i}, \bar{p}\right) \ln \left(\frac{\psi_{3 i}\left(u_{i}\left(t-\tau_{5 i}\right)\right)}{\psi_{3 i}\left(u_{i}\right)}\right)\right]+\sum_{i=1}^{2} \rho_{i} \lambda_{i} \chi_{i}\left(\bar{s}_{i}, \bar{p}\right)
\end{aligned}
$$




$$
\begin{aligned}
& -\sum_{i=1}^{2} \rho_{i}\left(\ell_{1 i} \lambda_{1 i} e^{-\mu_{1 i} \tau_{1 i}}+\ell_{2 i} \lambda_{2 i} e^{-\mu_{2 i} \tau_{2 i}}\right) \chi_{i}\left(\bar{s}_{i}, \bar{p}\right) \frac{\psi_{2 i}\left(y_{i}\left(t-\tau_{4 i}\right)\right) \psi_{41}(\bar{p})}{\psi_{2 i}\left(\bar{y}_{i}\right) \psi_{41}(p)} \\
& -\sum_{i=1}^{2} \rho_{i} \ell_{3 i} \lambda_{3 i} e^{-\mu_{3 i} \tau_{3 i}} \chi_{i}\left(\bar{s}_{i}, \bar{p}\right) \frac{\psi_{3 i}\left(u_{i}\left(t-\tau_{5 i}\right)\right) \psi_{41}(\bar{p})}{\psi_{3 i}\left(\bar{u}_{i}\right) \psi_{41}(p)} .
\end{aligned}
$$

By the equalities (1.15) with $\hat{s}_{i}=\bar{s}_{i}, \hat{w}_{i}=\bar{w}_{i}, \hat{y}_{i}=\bar{y}_{i}, \hat{u}_{i}=\bar{u}_{i}$, and $\hat{p}=\bar{p}$, we can get

$$
\begin{aligned}
& \frac{d V_{2}}{d t}=\sum_{i=1}^{2} \rho_{i}\left[\left(\pi_{i}\left(s_{i}\right)-\pi_{i}\left(\bar{s}_{i}\right)\right)\left(1-\frac{\chi_{i}\left(\bar{s}_{i}, \bar{p}\right)}{\chi_{i}\left(s_{i}, \bar{p}\right)}\right)\right. \\
& +\lambda_{i} \chi_{i}\left(\bar{s}_{i}, \bar{p}\right)\left(\frac{\chi_{i}\left(s_{i}, p\right)}{\chi_{i}\left(s_{i}, \bar{p}\right)}-\frac{\psi_{41}(p)}{\psi_{41}(\bar{p})}-1+\frac{\psi_{41}(p) \chi_{i}\left(s_{i}, \bar{p}\right)}{\psi_{41}(\bar{p}) \chi_{i}\left(s_{i}, p\right)}\right) \\
& -\lambda_{i} \chi_{i}\left(\bar{s}_{i}, \bar{p}\right)\left(\frac{\chi_{i}\left(\bar{s}_{i}, \bar{p}\right)}{\chi_{i}\left(s_{i}, \bar{p}\right)}-1-\ln \left(\frac{\chi_{i}\left(\bar{s}_{i}, \bar{p}\right)}{\chi_{i}\left(s_{i}, \bar{p}\right)}\right)\right) \\
& -\lambda_{i} \chi_{i}\left(\bar{s}_{i}, \bar{p}\right)\left(\frac{\psi_{41}(p) \chi_{i}\left(s_{i}, \bar{p}\right)}{\psi_{41}(\bar{p}) \chi_{i}\left(s_{i}, p\right)}-1-\ln \left(\frac{\psi_{41}(p) \chi_{i}\left(s_{i}, \bar{p}\right)}{\psi_{41}(\bar{p}) \chi\left(s_{i}, p\right)}\right)\right) \\
& -\ell_{1 i} \lambda_{1 i} \chi_{i}\left(\bar{s}_{i}, \bar{p}\right) e^{-\mu_{1 i} \tau_{1 i}}\left(\frac{\psi_{1 i}\left(\bar{w}_{i}\right) \chi_{i}\left(s_{i}\left(t-\tau_{1 i}\right), p\left(t-\tau_{1 i}\right)\right)}{\psi_{1 i}\left(w_{i}\right) \chi_{i}\left(\bar{s}_{i}, \bar{p}\right)}\right. \\
& \left.-1-\ln \left(\frac{\psi_{1 i}\left(\bar{w}_{i}\right) \chi_{i}\left(s_{i}\left(t-\tau_{1 i}\right), p\left(t-\tau_{1 i}\right)\right)}{\psi_{1 i}\left(w_{i}\right) \chi_{i}\left(\bar{s}_{i}, \bar{p}\right)}\right)\right) \\
& -\ell_{2 i} \lambda_{2 i} \chi_{i}\left(\bar{s}_{i}, \bar{p}\right) e^{-\mu_{2 i} \tau_{2 i}}\left(\frac{\psi_{2 i}\left(\bar{y}_{i}\right) \chi_{i}\left(s_{i}\left(t-\tau_{2 i}\right), p\left(t-\tau_{2 i}\right)\right)}{\psi_{2 i}\left(y_{i}\right) \chi_{i}\left(\bar{s}_{i}, \bar{p}\right)}\right. \\
& \left.-1-\ln \left(\frac{\psi_{2 i}\left(\bar{y}_{i}\right) \chi_{i}\left(s_{i}\left(t-\tau_{2 i}\right), p\left(t-\tau_{2 i}\right)\right)}{\psi_{2 i}\left(y_{i}\right) \chi_{i}\left(\bar{s}_{i}, \bar{p}\right)}\right)\right) \\
& -\ell_{3 i} \lambda_{3 i} \chi_{i}\left(\bar{s}_{i}, \bar{p}\right) e^{-\mu_{3 i} \tau_{3 i}}\left(\frac{\psi_{3 i}\left(\bar{u}_{i}\right) \chi_{i}\left(s_{i}\left(t-\tau_{3 i}\right), p\left(t-\tau_{3 i}\right)\right)}{\psi_{3 i}\left(u_{i}\right) \chi_{i}\left(\bar{s}_{i}, \bar{p}\right)}\right. \\
& \left.-1-\ln \left(\frac{\psi_{3 i}\left(\bar{u}_{i}\right) \chi_{i}\left(s_{i}\left(t-\tau_{3 i}\right), p\left(t-\tau_{3 i}\right)\right)}{\psi_{3 i}\left(u_{i}\right) \chi_{i}\left(\bar{s}_{i}, \bar{p}\right)}\right)\right) \\
& -\ell_{1 i} \lambda_{1 i} e^{-\mu_{1 i} \tau_{1 i}} \chi_{i}\left(\bar{s}_{i}, \bar{p}\right)\left(\frac{\psi_{2 i}\left(\bar{y}_{i}\right) \psi_{1 i}\left(w_{i}\right)}{\psi_{2 i}\left(y_{i}\right) \psi_{1 i}\left(\bar{w}_{i}\right)}-1-\ln \left(\frac{\psi_{2 i}\left(\bar{y}_{i}\right) \psi_{1 i}\left(w_{i}\right)}{\psi_{2 i}\left(y_{i}\right) \psi_{1 i}\left(\bar{w}_{i}\right)}\right)\right) \\
& -\left(\ell_{1 i} \lambda_{1 i} e^{-\mu_{1 i} \tau_{1 i}}+\ell_{2 i} \lambda_{2 i} e^{-\mu_{2 i} \tau_{2 i}}\right) \chi_{i}\left(\bar{s}_{i}, \bar{p}\right)\left(\frac{\psi_{2 i}\left(y_{i}\left(t-\tau_{4 i}\right)\right) \psi_{41}(\bar{p})}{\psi_{2 i}\left(\bar{y}_{i}\right) \psi_{41}(p)}\right. \\
& \left.-1-\ln \left(\frac{\psi_{2 i}\left(y_{i}\left(t-\tau_{4 i}\right)\right) \psi_{41}(\bar{p})}{\psi_{2 i}\left(\bar{y}_{i}\right) \psi_{41}(p)}\right)\right) \\
& \left.-\ell_{3 i} \lambda_{3 i} e^{-\mu_{3 i} \tau_{3 i}} \chi_{i}\left(\bar{s}_{i}, \bar{p}\right)\left(\frac{\psi_{3 i}\left(u_{i}\left(t-\tau_{5 i}\right)\right) \psi_{41}(\bar{p})}{\psi_{3 i}\left(\bar{u}_{i}\right) \psi_{41}(p)}-1-\ln \left(\frac{\psi_{3 i}\left(u_{i}\left(t-\tau_{5 i}\right)\right) \psi_{41}(\bar{p})}{\psi_{3 i}\left(\bar{u}_{i}\right) \psi_{41}(p)}\right)\right)\right] .
\end{aligned}
$$

Eq. (2.10) becomes

$$
\begin{aligned}
\frac{d V_{2}}{d t}= & \sum_{i=1}^{2} \rho_{i}\left[\left(\pi_{i}\left(s_{i}\right)-\pi_{i}\left(\bar{s}_{i}\right)\right)\left(1-\frac{\chi_{i}\left(\bar{s}_{i}, \bar{p}\right)}{\chi_{i}\left(s_{i}, \bar{p}\right)}\right)+\lambda_{i} \chi_{i}\left(\bar{s}_{i}, \bar{p}\right)\left(\frac{\chi_{i}\left(s_{i}, p\right)}{\chi_{i}\left(s_{i}, \bar{p}\right)}-\frac{\psi_{41}(p)}{\psi_{41}(\bar{p})}\right)\left(1-\frac{\chi_{i}\left(s_{i}, \bar{p}\right)}{\chi_{i}\left(s_{i}, p\right)}\right)\right. \\
& -\lambda_{i} \chi_{i}\left(\bar{s}_{i}, \bar{p}\right)\left[F\left(\frac{\chi_{i}\left(\bar{s}_{i}, \bar{p}\right)}{\chi_{i}\left(s_{i}, \bar{p}\right)}\right)+F\left(\frac{\psi_{41}(p) \chi_{i}\left(s_{i}, \bar{p}\right)}{\psi_{41}(\bar{p}) \chi_{i}\left(s_{i}, p\right)}\right)\right]-\ell_{1 i} \lambda_{1 i} e^{-\mu_{1 i} \tau_{1 i}} \chi_{i}\left(\bar{s}_{i}, \bar{p}\right) F\left(\frac{\psi_{2 i}\left(\bar{y}_{i}\right) \psi_{1 i}\left(w_{i}\right)}{\psi_{2 i}\left(y_{i}\right) \psi_{1 i}\left(\bar{w}_{i}\right)}\right) \\
& -\ell_{1 i} \lambda_{1 i} \chi_{i}\left(\bar{s}_{i}, \bar{p}\right) e^{-\mu_{1 i} \tau_{1 i} F}\left(\frac{\psi_{1 i}\left(\bar{w}_{i}\right) \chi_{i}\left(s_{i}\left(t-\tau_{1 i}\right), p\left(t-\tau_{1 i}\right)\right)}{\psi_{1 i}\left(w_{i}\right) \chi_{i}\left(\bar{s}_{i}, \bar{p}\right)}\right) \\
& -\ell_{2 i} \lambda_{2 i} \chi_{i}\left(\bar{s}_{i}, \bar{p}\right) e^{-\mu_{2 i} \tau_{2 i} F}\left(\frac{\psi_{2 i}\left(\bar{y}_{i}\right) \chi_{i}\left(s_{i}\left(t-\tau_{2 i}\right), p\left(t-\tau_{2 i}\right)\right)}{\psi_{2 i}\left(y_{i}\right) \chi_{i}\left(\bar{s}_{i}, \bar{p}\right)}\right) \\
& -\ell_{3 i} \lambda_{3 i} \chi_{i}\left(\bar{s}_{i}, \bar{p}\right) e^{-\mu_{3 i} \tau_{3 i}} F\left(\frac{\psi_{3 i}\left(\bar{u}_{i}\right) \chi_{i}\left(s_{i}\left(t-\tau_{3 i}\right), p\left(t-\tau_{3 i}\right)\right)}{\psi_{3 i}\left(u_{i}\right) \chi_{i}\left(\bar{s}_{i}, \bar{p}\right)}\right)
\end{aligned}
$$




$$
\begin{aligned}
& -\left(\ell_{1 i} \lambda_{1 i} e^{-\mu_{1 i} \tau_{1 i}}+\ell_{2 i} \lambda_{2 i} e^{-\mu_{2 i} \tau_{2 i}}\right) \chi_{i}\left(\bar{s}_{i}, \bar{p}\right) F\left(\frac{\psi_{2 i}\left(y_{i}\left(t-\tau_{4 i}\right)\right) \psi_{41}(\bar{p})}{\psi_{2 i}\left(\bar{y}_{i}\right) \psi_{4}(p)}\right) \\
& \left.-\ell_{3 i} \lambda_{3 i} e^{-\mu_{3 i} \tau_{3 i}} \chi_{i}\left(\bar{s}_{i}, \bar{p}\right) F\left(\frac{\psi_{3 i}\left(u_{i}\left(t-\tau_{5 i}\right)\right) \psi_{41}(\bar{p})}{\psi_{3 i}\left(\bar{u}_{i}\right) \psi_{41}(p)}\right)\right] .
\end{aligned}
$$

According to $\mathrm{H} 1, \mathrm{H} 2$, and $\mathrm{H} 4$ we get $\frac{d V_{2}}{\mathrm{dt}} \leqslant 0$ and $\frac{d V_{2}}{d t}=0$ at $\Pi_{2}$. LIP implies that $\Pi_{2}$ is GAS.

\section{Numerical simulations}

We now perform some computer simulations on the following application:

$$
\begin{aligned}
\dot{s}_{1}(t)= & \rho_{1}-\delta_{1} s_{1}(t)+B s_{1}(t)\left(1-\frac{s_{1}(t)}{s_{\max }}\right)-\frac{\left(1-\varepsilon_{1}\right) \bar{\lambda}_{1} s_{1}(t) p(t)}{1+\vartheta_{1} p(t)}, \\
\dot{s}_{2}(t)= & \rho_{2}-\delta_{2} s_{2}(t)-\frac{\left(1-f \varepsilon_{1}\right) \bar{\lambda}_{2} s_{2}(t) p(t)}{1+\vartheta_{2} p(t)}, \\
\dot{w}_{1}(t)= & \frac{\left(1-\varepsilon_{1}\right) \bar{\lambda}_{11} e^{-\mu_{11} \tau_{11}} s_{1}\left(t-\tau_{11}\right) p\left(t-\tau_{11}\right)}{1+\vartheta_{1} p\left(t-\tau_{11}\right)}-\left(\alpha_{1}+\beta_{1}\right) w_{1}(t), \\
\dot{w}_{2}(t)= & \frac{\left(1-f \varepsilon_{1}\right) \bar{\lambda}_{12} e^{-\mu_{12} \tau_{12}} s_{2}\left(t-\tau_{12}\right) p\left(t-\tau_{12}\right)}{1+\vartheta_{2} p\left(t-\tau_{12}\right)}-\left(\alpha_{2}+\beta_{2}\right) w_{2}(t) \\
\dot{y}_{1}(t)= & \frac{\left(1-\varepsilon_{1}\right) \bar{\lambda}_{21} e^{-\mu_{21} \tau_{21}} s_{1}\left(t-\tau_{21}\right) p\left(t-\tau_{21}\right)}{1+\vartheta_{1} p\left(t-\tau_{21}\right)}+\alpha_{1} w_{1}(t)-\eta_{1} y_{1}(t), \\
\dot{y}_{2}(t)= & \frac{\left(1-f \varepsilon_{1}\right) \bar{\lambda}_{22} e^{-\mu_{22} \tau_{22} s_{2}\left(t-\tau_{22}\right) p\left(t-\tau_{22}\right)}}{1+\vartheta_{2} p\left(t-\tau_{22}\right)}+\alpha_{2} w_{2}(t)-\eta_{2} y_{2}(t), \\
\dot{u}_{1}(t)= & \frac{\left(1-\varepsilon_{1}\right) \bar{\lambda}_{31} e^{-\mu_{31} \tau_{31}} s_{1}\left(t-\tau_{31}\right) p\left(t-\tau_{31}\right)}{1+\vartheta_{1} p\left(t-\tau_{31}\right)}-v_{1} u_{1}(t), \\
\dot{u}_{2}(t)= & \frac{\left(1-f \varepsilon_{1}\right) \bar{\lambda}_{32} e^{-\mu_{32} \tau_{32}} s_{2}\left(t-\tau_{32}\right) p\left(t-\tau_{32}\right)}{1+\vartheta_{2} p\left(t-\tau_{32}\right)}-v_{2} u_{2}(t) \\
\dot{p}(t)= & \left(1-\varepsilon_{2}\right) \bar{N}_{1} \eta_{1} e^{-\mu_{41} \tau_{41}} y_{1}\left(t-\tau_{41}\right)+\left(1-h \varepsilon_{2}\right) \overline{N_{2}} \eta_{2} e^{-\mu_{42} \tau_{42}} y_{2}\left(t-\tau_{42}\right) \\
& +\left(1-\varepsilon_{2}\right) \overline{M_{1}} v_{1} e^{-\mu_{51} \tau_{51}} u_{1}\left(t-\tau_{51}\right)+\left(1-h \varepsilon_{2}\right) \overline{M_{2}} v_{2} e^{-\mu_{52} \tau_{52} u_{2}\left(t-\tau_{52}\right)} \\
& -g p(t)-\mu p(t) x(t) \\
\dot{x}(t)= & r p(t) x(t)-\omega x(t)
\end{aligned}
$$

where $\mathrm{B}<\delta_{1}$. In this application, we consider the following specific forms of the general functions:

$$
\begin{aligned}
\pi_{1}\left(s_{1}(t)\right) & =\rho_{1}-\delta_{1} s_{1}(t)+B s_{1}(t)\left(1-\frac{s_{1}(t)}{s_{\max }}\right), & \pi_{2}\left(s_{2}(t)\right) & =\rho_{2}-\delta_{2} s_{2}(t), \\
\chi_{i}\left(s_{i}(t), p(t)\right) & =\frac{s_{i}(t) p(t)}{1+\vartheta_{i} p(t)}, & \psi_{j i}(\theta) & =\theta, j=1, \ldots, 4, i=1,2 .
\end{aligned}
$$

First we verify hypotheses H1-H4 for the chosen forms, then we solve the system using MATLAB. Clearly, $\pi_{\mathfrak{i}}(0)=\rho_{\mathfrak{i}}>0$ and $\pi_{\mathfrak{i}}\left(s_{i}^{0}\right)=0$, where

$$
s_{1}^{0}=\frac{s_{\max }}{2 B}\left(B-\delta_{1}+\sqrt{\left(B-\delta_{1}\right)^{2}+\frac{4 \rho_{1} B}{s_{\max }}}\right), \quad s_{2}^{0}=\frac{\rho_{2}}{\delta_{2}} .
$$

We have

$$
\pi_{1}^{\prime}\left(s_{1}\right)=-\delta_{1}+\mathrm{B}-\frac{2 \mathrm{~B} s_{1}}{s_{\max }}<0, \quad \pi_{2}^{\prime}\left(s_{2}\right)=-\delta_{2}<0 .
$$

Clearly, $\pi_{i}\left(s_{i}\right)>0$ for $s_{i} \in\left[0, s_{i}^{0}\right)$ and

$$
\pi_{1}\left(s_{1}\right)=\rho_{1}-\left(\delta_{1}-B\right) s_{1}-B \frac{s_{1}^{2}}{s_{\max }} \leqslant \rho_{1}-\left(\delta_{1}-B\right) s_{1}, \quad \pi_{2}\left(s_{2}\right)=\rho_{2}-\delta_{2} s_{2} .
$$


Then $\mathrm{H} 1$ is satisfied. We also have $\chi_{\mathfrak{i}}\left(s_{i}, p\right)>0, \chi_{\mathfrak{i}}(0, p)=\chi\left(s_{i}, 0\right)=0$ for $s_{i}, p \in(0, \infty)$, and

$$
\frac{\partial \chi_{i}\left(s_{i}, p\right)}{\partial s_{i}}=\frac{p}{\left(1+\vartheta_{i} p\right)^{\prime}}, \quad \frac{\partial \chi_{i}\left(s_{i}, p\right)}{\partial p}=\frac{s_{i}}{\left(1+\vartheta_{i} p\right)^{2}}, \quad \frac{\partial \chi_{i}\left(s_{i}, 0\right)}{\partial p}=s_{i} \text {. }
$$

Then, $\frac{\partial x_{i}\left(s_{i}, p\right)}{\partial s_{i}}>0, \frac{\partial x_{i}\left(s_{i}, p\right)}{\partial p}>0$, and $\frac{\partial x_{i}\left(s_{i}, 0\right)}{\partial p}>0$ for all $s_{i}, p \in(0, \infty)$. Therefore, H1 is satisfied. In addition

$$
\left(\frac{\partial x_{i}\left(s_{i}, 0\right)}{\partial p}\right)^{\prime}=1>0 \text { for all } s_{i}>0 .
$$

It follows that, $\mathrm{H} 2$ is satisfied. Clearly H3 holds true. Moreover,

$$
\frac{\partial}{\partial p}\left(\frac{\chi_{i}\left(s_{i}, p\right)}{\psi_{41}(p)}\right)=\frac{-\vartheta_{i} s}{\left(1+\vartheta_{i} p\right)^{2}}<0
$$

Therefore, $\mathrm{H} 4$ holds true and Theorems 2.1, 2.3, and 2.4 are applicable.The parameters $\mathrm{R}_{0}$ and $\mathrm{R}_{1}$ for this application are given by:

$$
\begin{aligned}
& R_{0}=\sum_{i=1}^{2} \frac{\left(\left\{N_{i} A_{i} e^{-\mu_{4 i} \tau_{4 i}}+M_{i} \lambda_{3 i} e^{-\mu_{3 i} \tau_{3 i}}\left(\alpha_{i}+\beta_{i}\right) e^{-\mu_{5 i} \tau_{5 i}}\right\}\right.}{g\left(\alpha_{i}+\beta_{i}\right)} s_{i}^{0}, \\
& R_{1}=\sum_{i=1}^{2} \frac{\left(\left\{N_{i} A_{i} e^{-\mu_{4 i} \tau_{4 i}}+M_{i} \lambda_{3 i} e^{-\mu_{3 i} \tau_{3 i}}\left(\alpha_{i}+\beta_{i}\right) e^{-\mu_{5 i} \tau_{5 i}}\right\}\right.}{g\left(\alpha_{i}+\beta_{i}\right)} \frac{\bar{s}_{i}}{1+\vartheta_{i} \bar{p}} \\
& A_{i}=\alpha_{i} \lambda_{1 i} e^{-\mu_{1 i} \tau_{1 i}}+\left(\alpha_{i}+\beta_{i}\right) \lambda_{2 i} e^{-\mu_{2 i} \tau_{2 i}} .
\end{aligned}
$$

Remark 3.1. There are several forms of the general function $\chi_{\mathfrak{i}}\left(s_{i}, p\right)$ where H1-H4 can be satisfied such as:

(i) Holling-type incidence $\chi_{i}\left(s_{i}, p\right)=\frac{s_{i} p}{1+\vartheta_{i} s_{i}}$;

(ii) Beddington-DeAngelis incidence $\chi_{i}\left(s_{i}, p\right)=\frac{s_{i} p}{1+\vartheta_{i} s_{i}+\sigma_{i} p}$;

(iii) Crowley-Martin incidence $\chi_{i}\left(s_{i}, p\right)=\frac{s_{i} p}{\left(1+\vartheta_{i} s_{i}\right)\left(1+\sigma_{i} p\right)}$;

(iv) Hill-type incidence $\chi_{i}\left(s_{i}, p\right)=\frac{s_{i}^{m} p}{\vartheta_{i}^{m}+s_{i}^{m}}$.

Now we are ready to perform some numerical simulations for system (3.1). The data of system (3.1) are

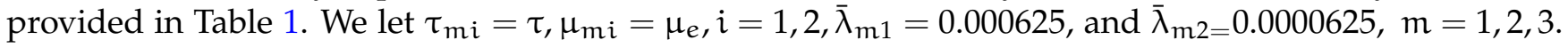

Table 1: The data of example (3.1).

\begin{tabular}{|c|c||c|c||c|c||c|c|}
\hline Parameter & Value & Parameter & Value & Parameter & Value & Parameter & Value \\
\hline$\rho_{1}$ & 10 & $\rho_{2}$ & 0.03198 & $\eta_{1}$ & 0.36 & $\eta_{2}$ & 0.03 \\
\hline$\delta_{1}$ & 0.01 & $\delta_{2}$ & 0.005 & $v_{1}$ & 0.031 & $\gamma_{2}$ & 0.01 \\
\hline $\mathrm{B}$ & 0.0001 & $\chi_{\max }$ & 1200 & $\mathrm{~g}$ & 3 & $\mu$ & 0.5 \\
\hline$\alpha_{1}$ & 0.2 & $\alpha_{2}$ & 0.01 & $\omega$ & 0.1 & $\mu_{e}$ & 1 \\
\hline$\beta_{1}$ & 0.02 & $\beta_{2}$ & 0.002 & $f$ & 0.5 & $\mathrm{~h}$ & 0.5 \\
\hline$\vartheta_{1}$ & 0.01 & $\vartheta_{2}$ & 0.002 & $\overline{\mathrm{N}}_{1}$ & 20 & $\overline{\mathrm{N}}_{2}$ & 5 \\
\hline $\bar{M}_{1}$ & 6 & $\bar{M}_{2}$ & 1 & $\varepsilon_{1}, \varepsilon_{2}$ & varied & $r, \tau$ & variad \\
\hline
\end{tabular}

\subsection{Stability of the steady states of the system}

To discuss our global results, we choose three different initial conditions:

IC1: $\left(s_{1}, s_{2}, w_{1}, w_{2}, y_{1}, y_{2}, u_{1}, u_{2}, p, x\right)(0)=(900,6,2,0.02,3,0.02,15,0.02,2,3)$;

IC2: $\left(s_{1}, s_{2}, w_{1}, w_{2}, y_{1}, y_{2}, u_{1}, u_{2}, p, x\right)(0)=(700,5,4,0.08,5,0.06,25,0.1,5,5)$;

IC3: $\left(s_{1}, s_{2}, w_{1}, w_{2}, y_{1}, y_{2}, u_{1}, u_{2}, p, x\right)(0)=(500,4,6,0.15,6.5,0.1,40,0.2,7,8)$; 
Let us address three cases for the parameters $\varepsilon_{1}, \varepsilon_{2}, \tau$, and $r$.

Case (I): Choose $\varepsilon_{1}=0.6, \varepsilon_{2}=0.8, \tau=1.0$, and $r=0.009$, which gives $R_{0}=0.1232<1$ and $R_{1}=0.0635<1$. Therefore, based on Theorem 2.1, the uninfected steady state $\Pi_{0}$ is GAS. As we can see from Figures 1-10 that the concentrations of the uninfected $\mathrm{CD}^{+} \mathrm{T}$ cells and macrophages are increased and approached their normal value before infection, that are, $s_{1}^{0}=1001.7, s_{2}^{0}=6.4$; while concentrations of the other compartments converge to zero for all the three initial conditions. As a result, the HIV is removed from the plasma.

Case (II): We take $\varepsilon_{1}=0.1, \varepsilon_{2}=0.2, \tau=0.5$, and $r=0.009$. For these values, $R_{1}=0.8910<1<R_{0}=$ 2.6577. Consequently, based on Theorem 2.3, the humoral-inactivated infection steady state $\Pi_{1}$ is GAS. Figures 1-10 confirm that the numerical results support the theoretical results presented in Theorem 2.3. It can be observed that, the variables of the model eventually converge to $\Pi_{1}=(443.186,4.960,5.143,0.121$, $6.0,0.089,36.497,0.145,8.088,0.0)$ for all the three initial conditions. This case corresponds to a chronic HIV infection in the absence of humoral immune response.

Case (III): $\varepsilon_{1}=0.1, \varepsilon_{2}=0.2, \tau=0.5$, and $r=0.08$. Then, we calculate $R_{0}=2.6577>1$ and $R_{1}=2.1744>1$. According to Theorem 2.4, the humoral-activated infection steady state $\Pi_{2}$ is GAS. We can see from Figures 1-10 that, there is a consistency between the numerical results and theoretical results of Theorem 2.4. The states of the system converge to $\Pi_{2}=(829.705,6.118,1.589,0.023,1.853,0.017,11.273,0.028,1.25,5.99)$ for all the three initial conditions. In this case the humoral immune response is activated and can control the disease.

\subsection{Effect of the time delay on the stability of the system}

Choosing $\varepsilon_{1}=\varepsilon_{2}=0$ and $r=0.08$, the initial conditions are considered to be $\left(s_{1}, s_{2}, w_{1}, w_{2}, y_{1}, y_{2}, u_{1}\right.$, $\left.u_{2}, p, x\right)(0)=(850,6.2,2,0.01,1.5,0.01,10,0.015,1,7)$. Figures 11-20 and Table 2 show the effect of the time delay parameter $\tau$ on the stability of $\Pi_{0}, \Pi_{1}$, and $\Pi_{2}$. Clearly, the parameter $\tau$ has similar effect as the drug efficacies parameters $\varepsilon_{1}$ and $\varepsilon_{2}$.

Table 2: The values of steady states, $R_{0}$ and $R_{1}$ for model (3.1) with different values of $\tau$.

\begin{tabular}{|c|c|c|c|}
\hline$\tau$ & steady states & $\mathrm{R}_{0}$ & $\mathrm{R}_{1}$ \\
\hline 0 & $\Pi_{2}=(814.16,6.11,2.86,0.04,3.33,0.03,20.27,0.05,1.25,38.42)$ & 9.22 & 7.40 \\
\hline 0.5 & $\Pi_{2}=(814.16,6.11,1.73,0.024,2.02,0.02,12.29,0.03,1.25,10.34)$ & 3.69 & 2.96 \\
\hline 1.0 & $\Pi_{2}=(814.28,6.11,1.05,1.23,1.19,0.011,7.45,0.02,1.25,0.01)$ & 1.54 & 1.24 \\
\hline 1.1 & $\Pi_{1}=(981.43,6.37,0.10,0.00,0.12,0.00,0.73,0.00,0.11,0)$ & 1.3 & 1.04 \\
\hline 1.2 & $\Pi_{0}=(1001.7,6.4,0,0,0,0,0,0,0,0)$ & 1.1 & 0.88 \\
\hline 1.5 & $\Pi_{0}=(1001.7,6.4,0,0,0,0,0,0,0,0)$ & 0.68 & 0.54 \\
\hline
\end{tabular}

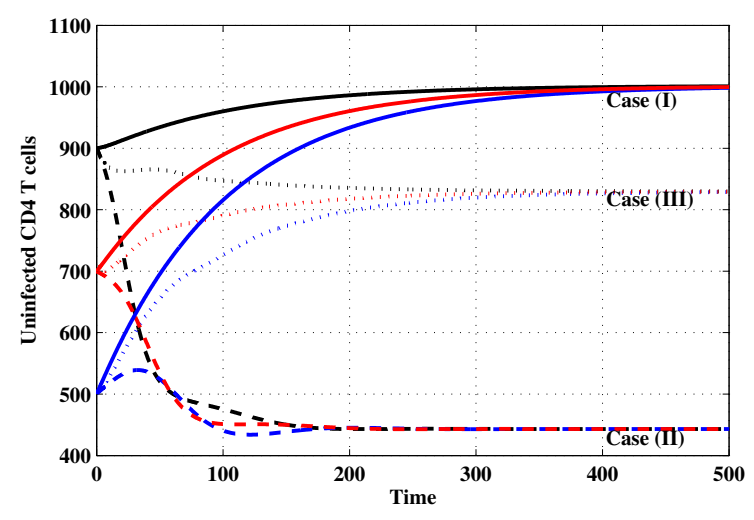

Figure 1: The concentration of uninfected $\mathrm{CD} 4^{+} \mathrm{T}$ cells.

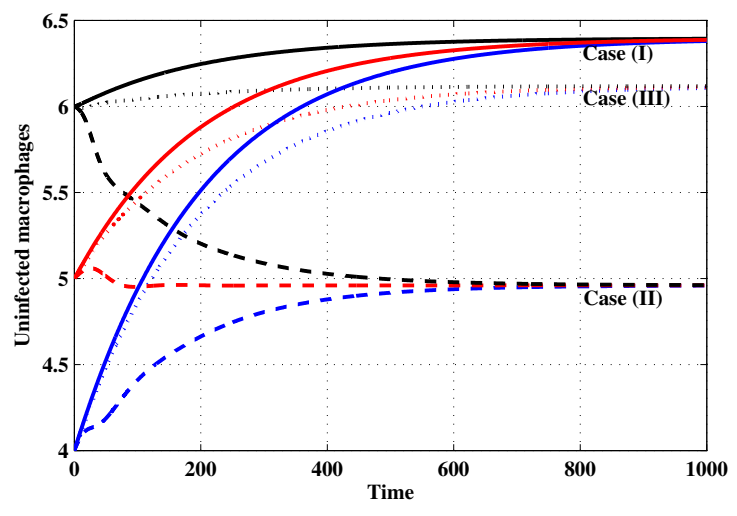

Figure 2: The concentration of uninfected macrophages. 


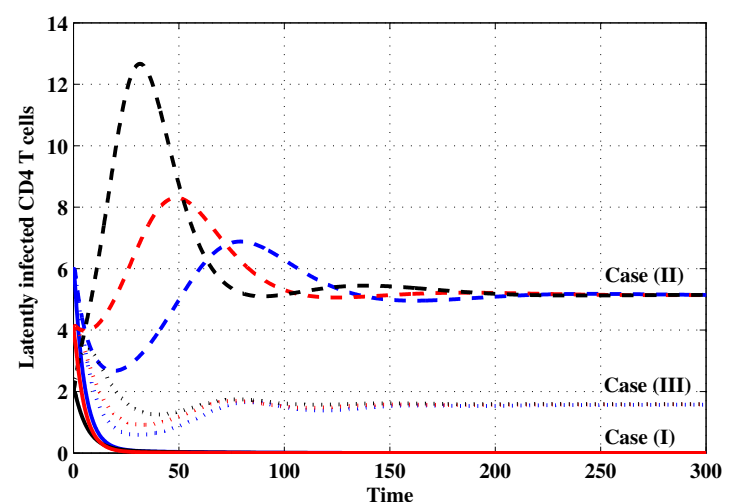

Figure 3: The concentration of latently infected $\mathrm{CD} 4^{+} \mathrm{T}$ cells.

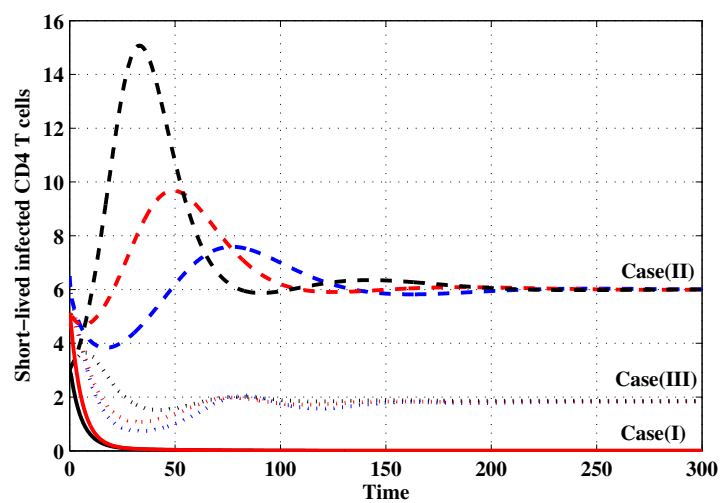

Figure 5: The concentration of short-lived productively infected $\mathrm{CD} 4^{+} \mathrm{T}$ cells.

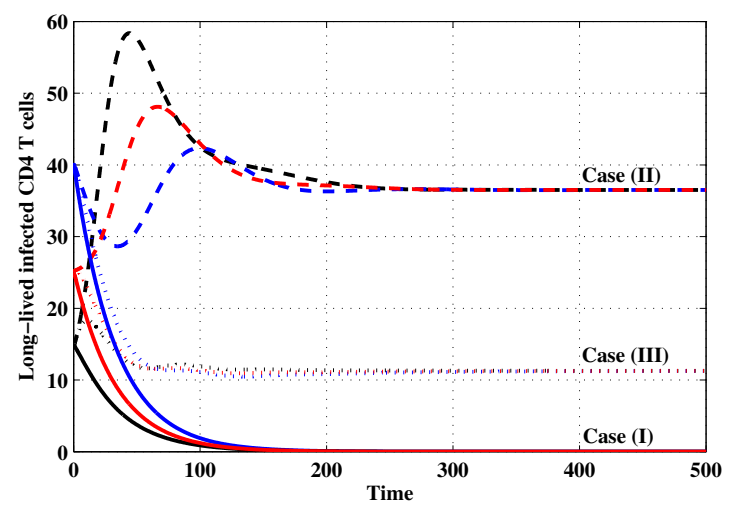

Figure 7: The concentration of long-lived productively infected $\mathrm{CD} 4^{+} \mathrm{T}$ cells.

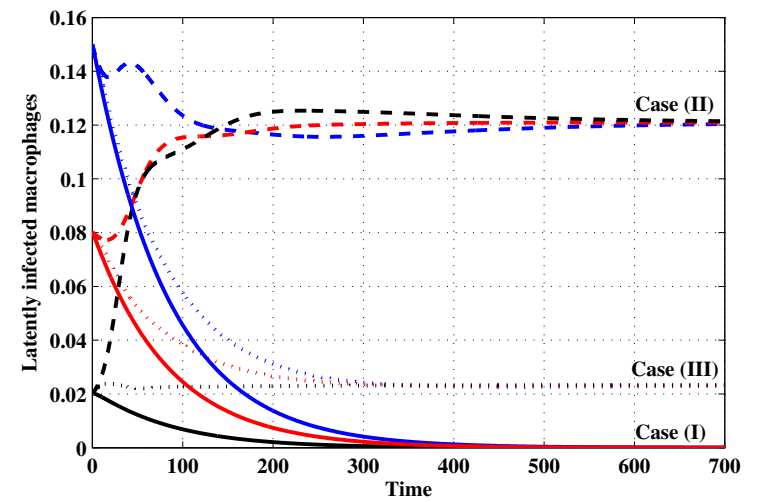

Figure 4: The concentration of latently infected macrophages.

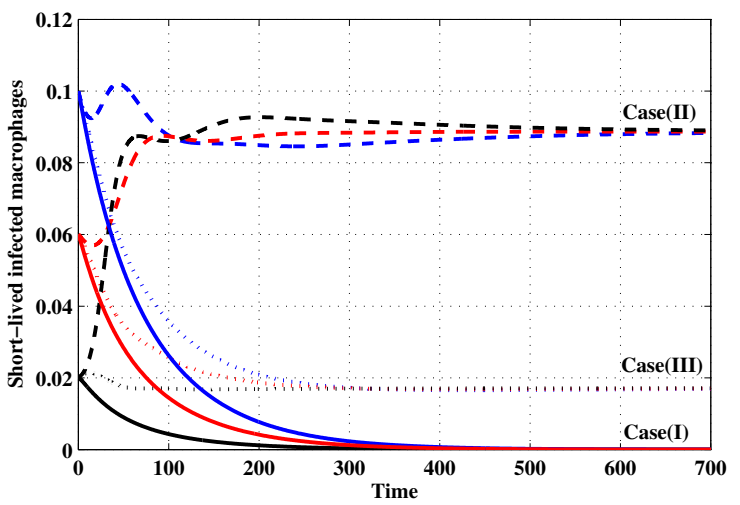

Figure 6: The concentration of short-lived productively infected macrophages.

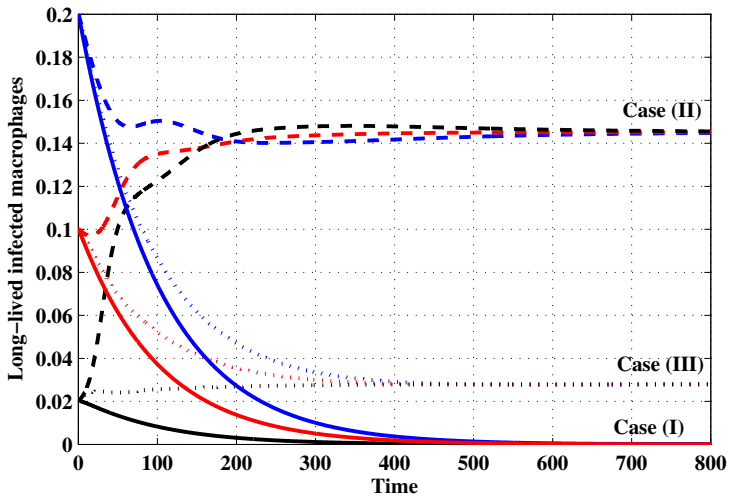

Figure 8: The concentration of long-lived productively infected macrophages. 


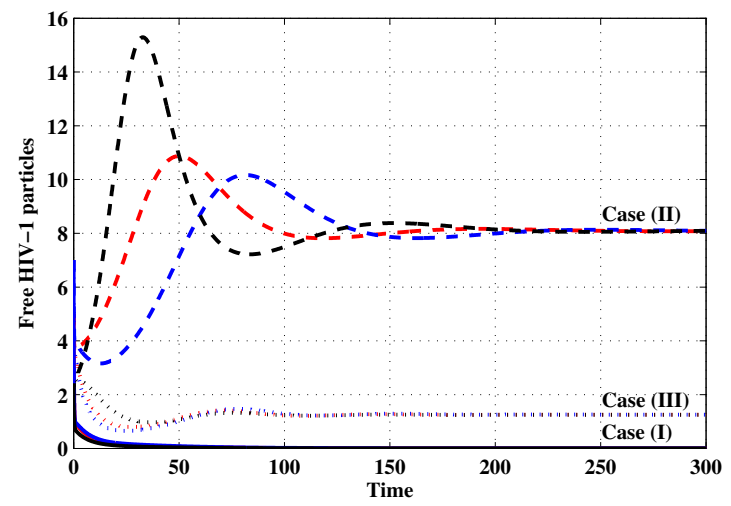

Figure 9: The concentration of free virus particles.

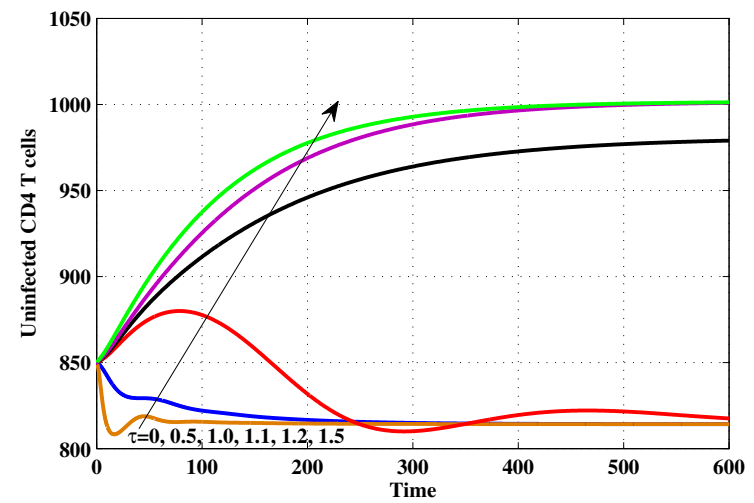

Figure 11: The concentration of uninfected CD4 ${ }^{+} \mathrm{T}$ cells.

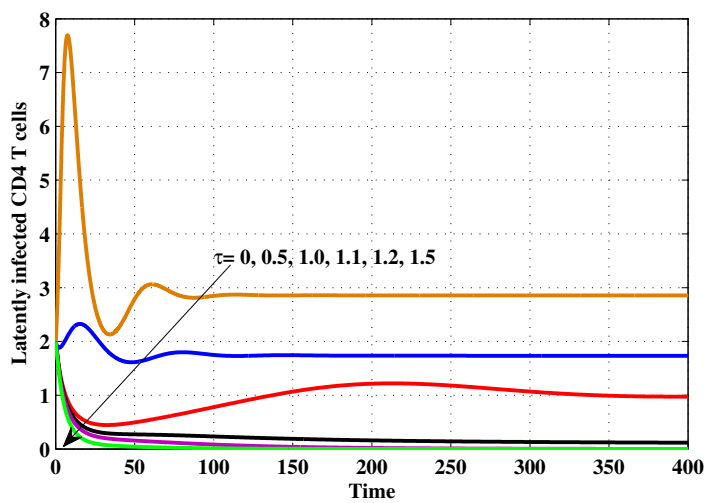

Figure 13: The concentration of latently infected $\mathrm{CD} 4^{+} \mathrm{T}$ cells.

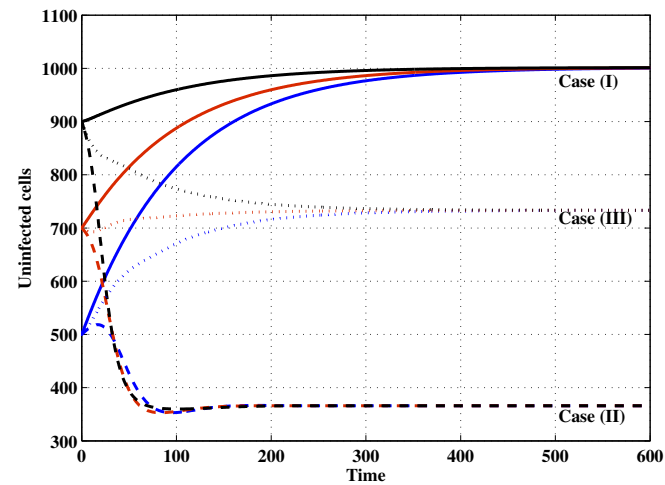

Figure 10: The concentration of B cells.

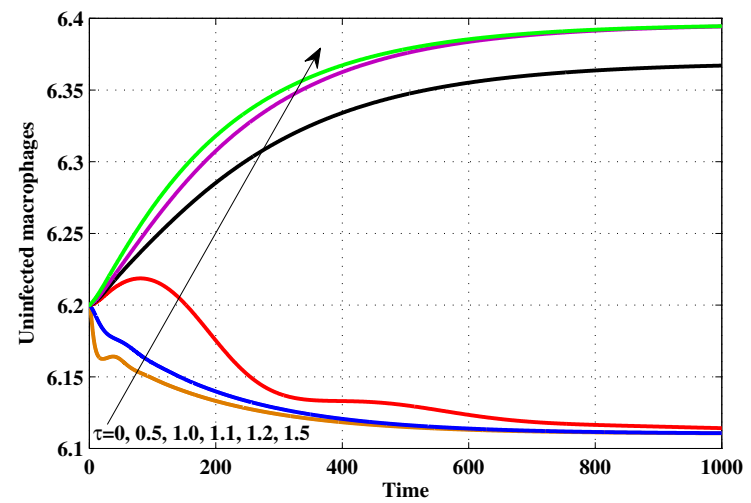

Figure 12: The concentration of uninfected macrophages.

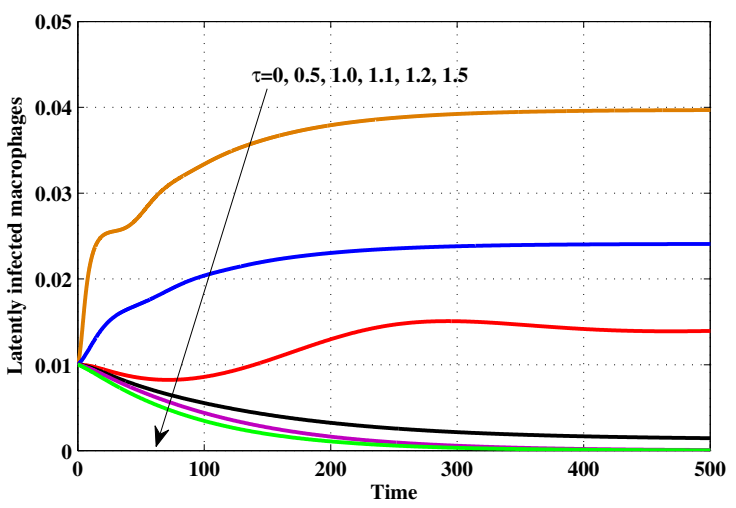

Figure 14: The concentration of latently infected macrophages. 


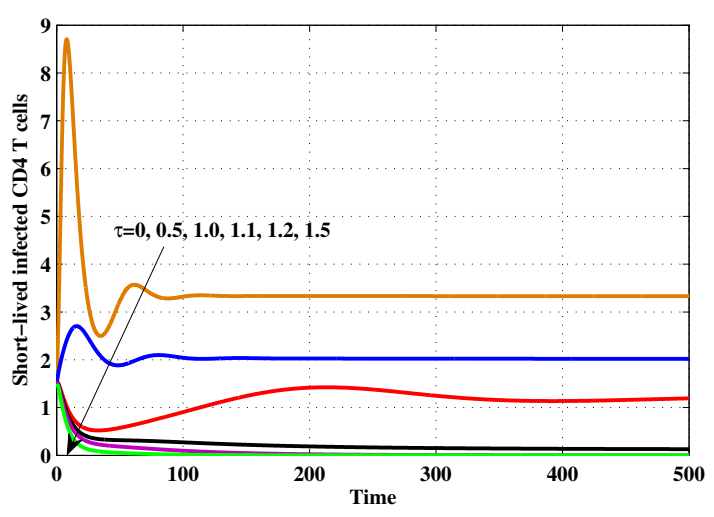

Figure 15: The concentration of short-lived productively infected $\mathrm{CD} 4^{+} \mathrm{T}$ cells.

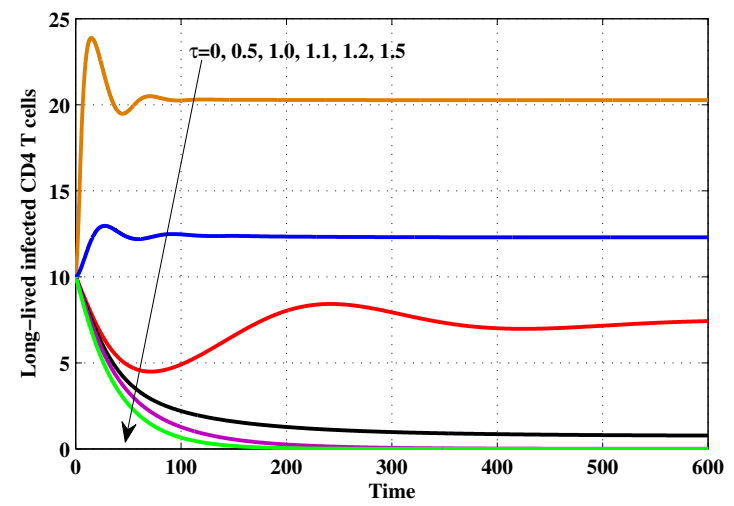

Figure 17: The concentration of long-lived productively infected $\mathrm{CD} 4^{+} \mathrm{T}$ cells.

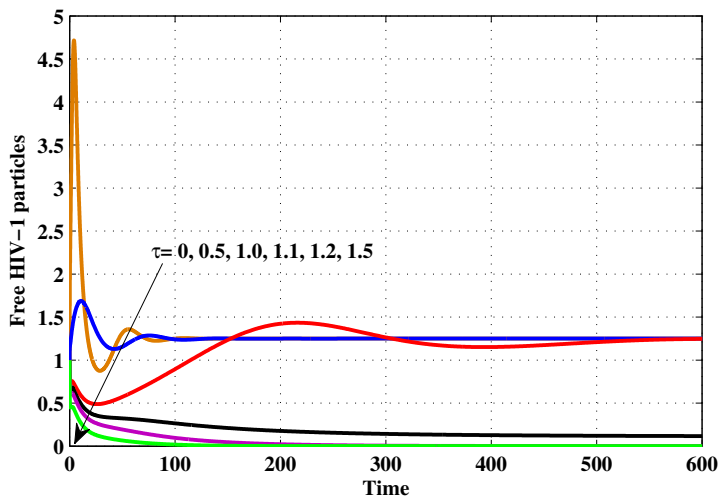

Figure 19: The concentration of free virus particles.

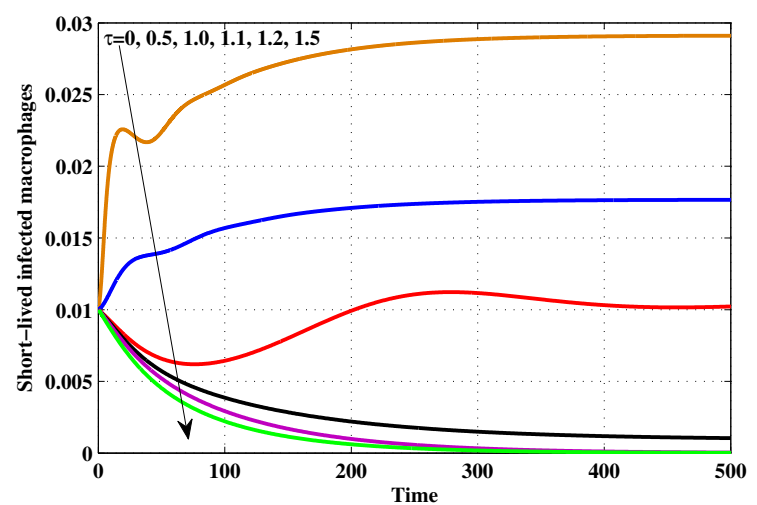

Figure 16: The concentration of short-lived productively infected macrophages.

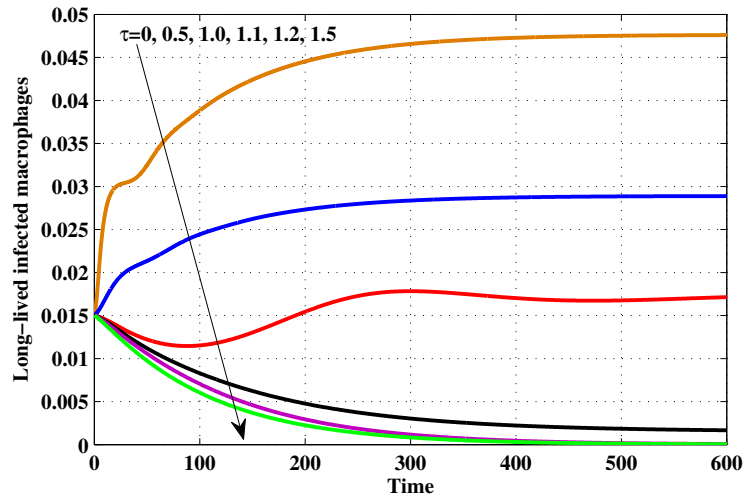

Figure 18: The concentration of long-lived productively infected macrophages.

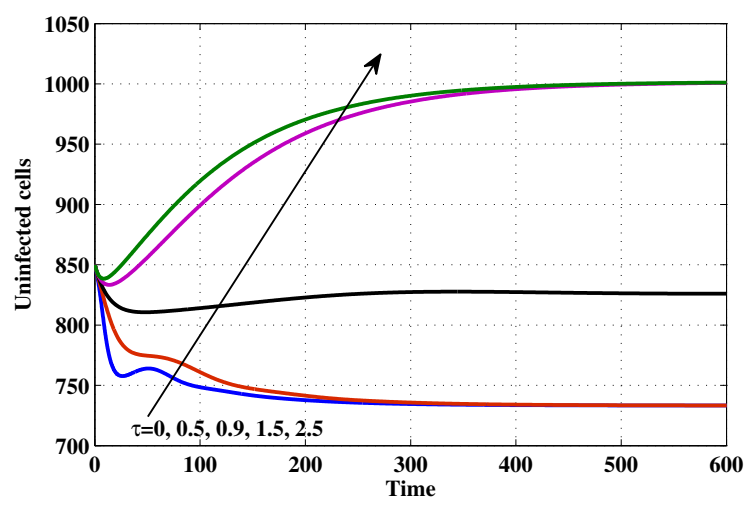

Figure 20: The concentration of B cells. 


\section{References}

[1] J. N. Blankson, D. Persaud, R. F. Siliciano, The challenge of viral reservoirs in HIV-1 infection, Annu. Rev. Med., 53 (2002), 557-593. 1

[2] B. Buonomo, C. Vargas-De-León, Global stability for an HIV-1 infection model including an eclipse stage of infected cells, J. Math. Anal. Appl., 385 (2012), 709-720. 1

[3] D. S. Callaway, A. S. Perelson, HIV-1 infection and low steady state viral loads, Bull. Math. Biol., 64 (2002), 29-64. 1

[4] R. V. Culshaw, S. Ruan, A delay-differential equation model of HIV infection of CD4 ${ }^{+}$T-cells, Math. Biosci., 165 (2000), 27-39.

[5] R. V. Culshaw, S. Ruan, G. Webb, A mathematical model of cell-to-cell spread of HIV-1 that includes a time delay, J. Math. Biol., 46 (2003), 425-444.

[6] A. Dutta , P. K. Gupta, A mathematical model for transmission dynamics of HIV/AIDS with effect of weak CD4 ${ }^{+} \mathrm{T}$ cells, Chin. J. Phys., 56 (2018), 1045-1056.

[7] A. M. Elaiw, Global properties of a class of HIV models, Nonlinear Anal. Real World Appl., 11 (2010), $2253-2263$.

[8] A. M. Elaiw, Global properties of a class of virus infection models with multitarget cells, Nonlinear Dynam., 69 (2012), 423-435. 1

[9] A. M. Elaiw, A. A. Almatrafi, A. D. Hobiny, Effect of antibodies on pathogen dynamics with delays and two routes of infection, AIP Advances, 8 (2018), 25 pages. 1

[10] A. M. Elaiw, N. A. Almuallem, Global dynamics of delay-distributed HIV infection models with differential drug efficacy in cocirculating target cells, Math. Methods Appl. Sci., 39 (2016), 4-31. 1

[11] A. M. Elaiw, N. A. Almuallem, Global properties of delayed-HIV dynamics models with differential drug efficacy in cocirculating target cells, Appl. Math. Comput., 265 (2015), 1067-1089.

[12] A. M. Elaiw, N. H. AlShamrani, Global properties of nonlinear humoral immunity viral infection models, Int. J. Biomath., 8 (2015), 53 pages. 1

[13] A. M. Elaiw, N. H. AlShamrani, Global stability of humoral immunity virus dynamics models with nonlinear infection rate and removal, Nonlinear Anal. Real World Appl., 26 (2015), 161-190. 1

[14] A. M. Elaiw, N. H. AlShamrani, Stability of a general delay-distributed virus dynamics model with multi-staged infected progression and immune response, Math. Methods Appl. Sci., 40 (2017), 699-719. 1

[15] A. M. Elaiw, N. H. AlShamrani, Stability of an adaptive immunity pathogen dynamics model with latency and multiple delays, Math. Methods Appl. Sci., 36 (2018), 125-142. 1

[16] A. M. Elaiw, N. H. AlShamrani, A. S. Alofi, Stability of CTL immunity pathogen dynamics model with capsids and distributed delay, AIP Advances, 7 (2017), 24 pages. 1

[17] A. M. Elaiw, N. H. AlShamrani, K. Hattaf, Dynamical behaviors of a general humoral immunity viral infection model with distributed invasion and production, Int. J. Biomath., 10 (2017), 25 pages.

[18] A. M. Elaiw, S. A. Azoz, Global properties of a class of HIV infection models with Beddington-DeAngelis functional response, Math. Methods Appl. Sci., 36 (2013), 383-394. 1

[19] A. M. Elaiw, E. K. Elnahary, A. A. Raezah, Effect of cellular reservoirs and delays on the global dynamics of HIV, Adv. Difference Equ., 2018 (2018), 36 pages. 1

[20] A. M. Elaiw, I. A. Hassanien, S. A. Azoz, Global stability of HIV infection models with intracellular delays, J. Korean Math. Soc., 49 (2012), 779-794. 1

[21] A. M. Elaiw, A. A. Raezah, Stability of general virus dynamics models with both cellular and viral infections and delays, Math. Methods Appl. Sci., 40 (2017), 5863-5880.

[22] A. M. Elaiw, A. A. Raezah, A. S. Alofi, Effect of humoral immunity on HIV-1 dynamics with virus-to-target and infectedto-target infections, AIP Advances, 6 (2016), 16 pages.

[23] A. M. Elaiw, A. A. Raezah, A. S. Alofi, Stability of a general delayed virus dynamics model with humoral immunity and cellular infection, AIP Advances, 7 (2017), 21 pages.

[24] A. M. Elaiw,, A. A. Raezah, K. Hattaf, Stability of HIV-1 infection with saturated virus-target and infected-target incidences and CTL immune response, Int. J. Biomath., 10 (2017), 29 pages.

[25] L. Gibelli, A. Elaiw, M. A. Alghamdi, A. M. Althiabi, Heterogeneous population dynamics of active particles: Progression, mutations, and selection dynamics, Math. Models Methods Appl. Sci., 27 (2017), 617-640. 1

[26] J. K. Hale, S. M. Verduyn Lunel, Introduction to functional differential equations, Springer-Verlag, New York (1993). 1.1

[27] K. Hattaf, N. Yousfi, A generalized virus dynamics model with cell-to-cell transmission and cure rate, Adv. Difference Equ., 2016 (2016), 11 pages. 1

[28] G. Huang, Y. Takeuchi, W. Ma, Lyapunov functionals for delay differential equations model of viral infections, SIAM J. Appl. Math., 70 (2010), 2693-2708. 1

[29] A. Korobeinikov, Global properties of basic virus dynamics models, Bull. Math. Biol., 66 (2004), 879-883. 1

[30] B. Li, Y. M. Chen, X. J. Lu, S. Q. Liu, A delayed HIV-1 model with virus waning term, Math. Biosci. Eng., 13 (2016), 135-157. 1

[31] M. Y. Li, L. C. Wang, Backward bifurcation in a mathematical model for HIV infection in vivo with anti-retroviral treatment, Nonlinear Anal. Real World Appl., 17 (2014), 147-160. 
[32] P. W. Nelson, J. D. Murray, A. S. Perelson, A model of HIV-1 pathogenesis that includes an intracellular delay, Math. Biosci., 163 (2000), 201-215.

[33] M. A. Nowak, C. R. M. Bangham. Population dynamics of immune responses to persistent viruses, Science, 272 (1996), 74-79. 1

[34] A. S. Perelson, P. Essunger, Y. Cao, M. Vesanen, A. Hurley, K. Saksela, M. Markowitz, D. D. Ho, Decay characteristics of HIV-1 infected compartments during combination therapy, Nature, 387 (1997), 188-191. 1

[35] C. M. A. Pinto, A. R. M. Carvalho, A latency fractional order model for HIV dynamics, J. Comput. Appl. Math., 312 (2017), 240-256. 1

[36] L. C. Wang, M. Y. Li, Mathematical analysis of the global dynamics of a model for HIV infection of CD4 ${ }^{+}$T cells, Math. Biosci., 200 (2006), 44-57.

[37] J. P. Wang, Z. D. Teng, H. Miao, Global dynamics for discrete-time analog of viral infection model with nonlinear incidence and CTL immune response, Adv. Difference Equ., 2016 (2016), 19 pages. 1

[38] X. Yang, L. S. Chen, J. F. Chen, Permanence and positive periodic solution for the single-species nonautonomous delay diffusive models, Comput. Math. Appl., 32 (1996), 109-116. 1.1

[39] Y. Zhao, D. T. Dimitrov, H. Liu, Y. Kuang, Mathematical insights in evaluating state dependent effectiveness of HIV prevention interventions, Bull. Math. Biol., 75 (2013), 649-675. 1 